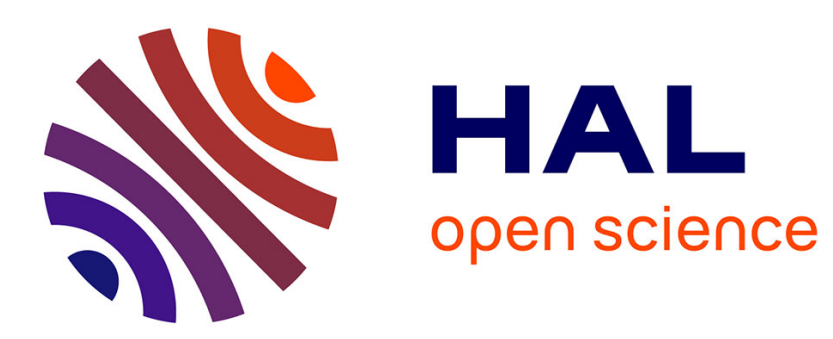

\title{
Linear non-normal energy amplification of harmonic and stochastic forcing in the turbulent channel flow \\ Yongyun Hwang, Carlo Cossu
}

\section{To cite this version:}

Yongyun Hwang, Carlo Cossu. Linear non-normal energy amplification of harmonic and stochastic forcing in the turbulent channel flow. Journal of Fluid Mechanics, 2010, 664 (December), pp.51-73. 10.1017/s0022112010003629 . hal-01020668

\section{HAL Id: hal-01020668}

https: / hal-polytechnique.archives-ouvertes.fr/hal-01020668

Submitted on 9 Jul 2014

HAL is a multi-disciplinary open access archive for the deposit and dissemination of scientific research documents, whether they are published or not. The documents may come from teaching and research institutions in France or abroad, or from public or private research centers.
L'archive ouverte pluridisciplinaire HAL, est destinée au dépôt et à la diffusion de documents scientifiques de niveau recherche, publiés ou non, émanant des établissements d'enseignement et de recherche français ou étrangers, des laboratoires publics ou privés. 


\title{
Linear non-normal energy amplification of harmonic and stochastic forcing in the turbulent channel flow
}

\author{
YONGYUN HWANG ${ }^{1} \dagger$ AND CARLO COSSU $\mathrm{C}^{2,3}$ \\ ${ }^{1}$ Laboratorie d'Hydrodynamique (LadHyX), CNRS-École Polytechnique, \\ F-91128 Palaiseau, France \\ ${ }^{2}$ CNRS-Institut de Mécanique des Fluides de Toulouse (IMFT), \\ Allée du Professeur Camille Soula, F-31400 Toulouse, France \\ ${ }^{3}$ Département de Mécanique, École Polytechnique, F-91128 Palaiseau, France \\ (Received 8 January 2010; revised 5 July 2010; accepted 5 July 2010; \\ first published online 22 September 2010)
}

The linear response to stochastic and optimal harmonic forcing of small coherent perturbations to the turbulent channel mean flow is computed for Reynolds numbers ranging from $R e_{\tau}=500$ to 20000 . Even though the turbulent mean flow is linearly stable, it is nevertheless able to sustain large amplifications by the forcing. The most amplified structures consist of streamwise-elongated streaks that are optimally forced by streamwise-elongated vortices. For streamwise-elongated structures, the mean energy amplification of the stochastic forcing is found to be, to a first approximation, inversely proportional to the forced spanwise wavenumber while it is inversely proportional to its square for optimal harmonic forcing in an intermediate spanwise wavenumber range. This scaling can be explicitly derived from the linearized equations under the assumptions of geometric similarity of the coherent perturbations and of logarithmic base flow. Deviations from this approximate power-law regime are apparent in the pre-multiplied energy amplification curves that reveal a strong influence of two different peaks. The dominant peak scales in outer units with the most amplified spanwise wavelength of $\lambda_{z} \approx 3.5 h$, while the secondary peak scales in wall units with the most amplified $\lambda_{z}^{+} \approx 80$. The associated optimal perturbations are almost independent of the Reynolds number when, respectively, scaled in outer and inner units. In the intermediate wavenumber range, the optimal perturbations are approximatively geometrically similar. Furthermore, the shape of the optimal perturbations issued from the initial value, the harmonic forcing and the stochastic forcing analyses are almost indistinguishable. The optimal streaks corresponding to the large-scale peak strongly penetrate into the inner layer, where their amplitude is proportional to the mean-flow profile. At the wavenumbers corresponding to the large-scale peak, the optimal amplifications of harmonic forcing are at least two orders of magnitude larger than the amplifications of the variance of stochastic forcing and both increase with the Reynolds number. This confirms the potential of the artificial forcing of optimal large-scale streaks for the flow control of wall-bounded turbulent flows.

Key words: turbulent boundary layers, turbulence theory 


\section{Introduction}

The understanding of the dynamics of wall-bounded turbulent flows is an interesting topic in its own right, but it is also relevant for applications ranging from flow control to atmospheric dynamics. For these applications, the high-Reynolds-number regime is of particular interest. In this regime, it is generally accepted that the dissipation of energy is associated with structures at very small scales, while there is much less consensus on the mechanisms by which energy is extracted from the mean flow and injected in the turbulent motions. In this respect, much attention is currently given to the dynamics of streamwise streaks which are narrow regions, elongated in the streamwise direction, of excess or deficit of streamwise velocity. There is a growing body of numerical and experimental evidence of the existence of streamwise streaks ranging from small to very large scales. It has been known for a while that streaky motions with a mean spanwise spacing of about 100 wall units exist in the buffer layer (Kline et al. 1967; Smith \& Metzler 1983). These streaky motions have been shown to be able to self-sustain even in the absence of outer-layer motions and to substantially contribute to the production of turbulent kinetic energy in the near-wall region (Jiménez \& Moin 1991; Jiménez \& Pinelli 1999). The self-sustained mechanism is now understood to be based on the strong amplification of the streaks from quasi-streamwise vortices followed by the breakdown of the streaks leading to the 'refuelling' of the quasi-streamwise vortices (Hamilton, Kim \& Waleffe 1995; Waleffe 1995; Schoppa \& Hussain 2002).

Streaky motions also exist at larger scales and have been observed recently in most wall-bounded flows such as plane Couette flow (Komminaho, Lundbladh \& Johansson 1996; Kitoh, Nakabayashi \& Nishimura 2005), pipes (Kim \& Adrian 1999; Guala, Hommema \& Adrian 2006), plane Poiseuille flow (Jiménez 1998; del Álamo \& Jiménez 2003; del Álamo et al. 2004) and flat-plate boundary layers (Tomkins \& Adrian 2003, 2005; Hutchins \& Marusic 2007a,b). These streaky motions, called 'very large-scale motion' or 'global mode' or 'superstructure', extend up to a few outer length units, $h$, in the spanwise direction, and they carry a significant amount of the turbulent kinetic energy and Reynolds stress in the outer region (Komminaho et al. 1996; Tomkins \& Adrian 2005; Guala et al. 2006). Moreover, the influence of these energetic structures is felt even in the buffer layer, leading to the modulation of the near-wall cycles (Hunt \& Morrison 2000; Hutchins \& Marusic 2007b; Mathis, Hutchins \& Marusic 2009).

The understanding of the physical mechanism by which energy could be extracted from the turbulent mean flow and, in particular, the mechanism leading to the amplification of the streaks, has long been elusive because the mean velocity profiles of the wall-bounded turbulent flows are linearly stable (Malkus 1956; Reynolds \& Tiederman 1967). However, in the more tractable case of laminar base flows, it has been shown that large shear can support the strong amplification of streamwise vortices into streamwise streaks via the lift-up effect (Moffatt 1967; Ellingsen \& Palm 1975; Landahl 1980, 1990). This amplification is associated with the non-normality of the linearized Navier-Stokes operator (for a review see e.g. Trefethen et al. 1993; Farrell \& Ioannou 1996; Schmid \& Henningson 2001). In the initial-value problem framework, of interest for the understanding of subcritical transition, the optimal amplification of streaks has been shown to be proportional to the square of the Reynolds number (Gustavsson 1991) and has been computed for virtually all the canonical laminar shear flows (Butler \& Farrell 1992; Reddy \& Henningson 1993; Schmid \& Henningson 1994). 
It is tempting to transpose the lift-up mechanism to the case of turbulent flows. In this case, however, one has to deal with the unsteadiness and randomness of the local shear promoting the lift-up. Progress has been made by considering the Reynolds-averaged Navier-Stokes equations linearized in the neighbourhood of the turbulent mean flow. These equations rule the dynamics of small-amplitude coherent perturbations, while all the effects of the other perturbations, which have a zero statistical average, are captured by the Reynolds shear-stress tensor. Reynolds \& Hussain (1972) have shown that a formulation where the Reynolds shear stress is modelled with an eddy viscosity is much better adapted to the description of the motion of small-amplitude coherent perturbations than the one where the Reynolds shear stress is simply neglected. Del Alamo \& Jiménez (2006) and Pujals et al. (2009) have adopted this modelling for the computation of the optimal temporal transient growths supported by the turbulent channel flow. They find that only streamwiseelongated structures are noticeably amplified. For these structures, at sufficiently high Reynolds numbers, two locally optimal spanwise wavelengths exist that, respectively, scale in outer and inner units. The global maximum growth is associated to the main peak at $\lambda_{z} \approx 4 h$, in fair agreement with the spanwise spacing of large-scale streaky motions in the outer region. The secondary peak of the growth was found at $\lambda_{z}^{+} \approx 100$, which corresponds well to the average spanwise spacing of the buffer-layer streaks. These results strongly suggest that a 'coherent lift-up' mechanism plays a crucial role in the selection of the spanwise scales of streaky structures in turbulent flows.

In turbulent flows, structures of a given scale are permanently forced by structures of different scales via nonlinear interactions. In this context, a modelling of the streaks' amplification based on the response to stochastic forcing would seem more appropriate than the optimal initial-value problem considered in previous investigations. Furthermore, in the perspective of flow control, it is also of interest to compute the optimal response of the turbulent flow to deterministic forcing. In the case of laminar flows the computation of the response to optimal harmonic (deterministic) forcing (Reddy \& Henningson 1993; Reddy, Schmid \& Henningson 1993; Trefethen et al. 1993) and to stochastic forcing (Farrell \& Ioannou 1993a,b, 1996; Bamieh \& Dahleh 2001; Jovanović \& Bamieh 2005) is routine, but this is not the case for turbulent flows.

In this study, we compute the response of the turbulent channel flow to both stochastic and optimal harmonic forcing for Reynolds numbers ranging from $R e_{\tau}=$ 500 to 20000 . We anticipate that the amplification of the forcing is found to always decrease with the scale of the forced structures, contrary to what has been found for the optimal growth of initial perturbations. In particular, we show that for streamwise uniform and geometrically similar structures living in the log layer, the energy amplification of stochastic forcing and of harmonic forcing scale like $\beta^{-1}$ and $\beta^{-2}$, respectively, where $\beta$ is their spanwise wavenumber. This scaling is actually found to approximatively apply to the numerical results in an intermediate wavenumber range. The use of pre-multiplied energy amplifications allows us then to analyse the deviations from the $\beta$-power-law behaviour and to identify the spanwise scales associated to the most amplified structures in the near wall and at large scales. Previous similar investigations have not detected the approximate $\beta$-power scaling of the amplifications of log-layer structures, either because the molecular viscosity instead of the turbulent eddy viscosity was used in the linearized equations (Farrell \& Ioannou 1998) or because a too low Reynolds number was considered (Hwang \& Cossu 2010). 
This paper is organized as follows: in $\S 2$, we briefly introduce the considered turbulent mean base flow, the associated eddy viscosity and the equations satisfied by small coherent perturbations to the base flow. We then recall the standard framework adopted for the computation of the response to stochastic forcing and for the optimal response to harmonic forcing. The amplifications of the forcing computed for $R e_{\tau}=10000$ are analysed in $\S 3$ and their dependence on the Reynolds number is detailed in $\S 4$. The scaling with the wavenumber of the amplification of geometrically similar log-layer structures is derived from the linear model in $\S 5$ with some of the details reported in the Appendix. The results are discussed in $\S 6$, while the main findings are summarized in $\S 7$.

\section{Background}

\subsection{Linear model for the evolution of small-amplitude coherent structures}

We consider a pressure-driven turbulent channel flow of incompressible viscous fluid with density $\rho$, kinematic viscosity $v$ and mean centreline velocity $U_{e}$ between two infinite parallel walls located at $y= \pm h$. Here, we denote streamwise, wall-normal and spanwise coordinates by $x, y$ and $z$, respectively. In the following, we will also use the distance from the wall scaled in wall (inner) units and defined as $y^{+}=(y+h) u_{\tau} / v$ when the lower wall is considered. Following Reynolds \& Hussain (1972), del Álamo \& Jiménez (2006) and Pujals et al. (2009) and others, we introduce the linearized equations for 'small coherent perturbations' in the presence of the total eddy viscosity $v_{T}(y)=v+v_{t}(y)$

$$
\begin{gathered}
\nabla \cdot \boldsymbol{u}=0, \\
\frac{\partial \boldsymbol{u}}{\partial t}+\nabla \boldsymbol{u} \cdot \boldsymbol{U}+\nabla \boldsymbol{U} \cdot \boldsymbol{u}=-\frac{1}{\rho} \nabla p+\nabla \cdot\left[v_{T}\left(\nabla \boldsymbol{u}+\nabla \boldsymbol{u}^{T}\right)\right]+\boldsymbol{f},
\end{gathered}
$$

where $\boldsymbol{U}$ is the base mean velocity, $\boldsymbol{u}=(u, v, w)$ and $p$ are the coherent perturbation velocity and pressure, respectively, and $\boldsymbol{f}=\left(f_{u}, f_{v}, f_{w}\right)$ is the forcing term. These equations can be derived from the Reynolds-averaged Navier-Stokes equations by the standard, if crude, modelling of the Reynolds stress term with an isotropic eddy viscosity and by a linearization near the base flow $\boldsymbol{U}$. The base flow should be understood as the turbulent mean flow obtained for $\boldsymbol{f}=\mathbf{0}$ in the absence of any statistically correlated initial condition. In that case, the temporal and horizontal statistical invariance of the mean solution gives $\boldsymbol{U}=\left(U_{b}(y), 0,0\right)$. In the following we will refer to 'coherent perturbation' as a perturbation $\boldsymbol{u}$ of the base flow $\boldsymbol{U}$ that has a non-zero ensemble average. Note that $\boldsymbol{u}$ does not need to be steady or spatially uniform. In an experiment or a direct numerical simulation (DNS) these coherent perturbations would typically be realized using artificial forcing (see e.g. Reynolds $\&$ Hussain 1972), ensuring that their spatial and temporal phases are always the same in each realization or by locating and tracking (with same phase) the same kind of event over multiple realizations. The linearized equations (2.1) apply to these coherent perturbations if their amplitude is sufficiently small, which does not seem too unreasonable. Indeed, the amplitudes of natural or artificially forced output streaks are typically not larger than $\approx O\left(10^{-1} U_{e}\right)$. Furthermore, as the input-output energy amplifications are typically large, the amplitude of the optimal input vortices needed to force streaks at the observed small amplitudes are even smaller. This supports the idea that an optimal coherent lift-up process mainly acts at small perturbation amplitude levels, where the linear approximation applies. 
For the total eddy viscosity, we adopt the semi-empirical expression proposed by Cess (1958), as reported by Reynolds \& Tiederman (1967):

$$
\nu_{T}(\eta)=\frac{v}{2}\left\{1+\frac{\kappa^{2} R e_{\tau}^{2}}{9}\left(1-\eta^{2}\right)^{2}\left(1+2 \eta^{2}\right)^{2} \times\left\{1-\exp \left[(|\eta|-1) R e_{\tau} / A\right]\right\}^{2}\right\}^{1 / 2}+\frac{v}{2},
$$

where $\eta=y / h$ and $R e_{\tau}=u_{\tau} h / v$ is the Reynolds number based on the friction velocity $u_{\tau}$. We set the von Kármán constant $\kappa=0.426$ and the constant $A=25.4$ as in del Alamo \& Jiménez (2006) and Pujals et al. (2009). Note that these values were obtained from fits based on a DNS performed at $R e_{\tau}=2000$ (Hoyas \& Jiménez 2006), thus they may not be reliable far from this Reynolds number. The mean velocity profile is obtained by integrating $\mathrm{d} U_{b}^{+} / \mathrm{d} \eta=-R e_{\tau} \eta / \nu_{T}^{+}(\eta)$, where $\nu_{T}^{+}=\nu_{T} / \nu$. Since the mean flow is homogeneous in the streamwise and spanwise directions, we consider the plane Fourier modes $\hat{\boldsymbol{u}}(y, t ; \alpha, \beta) \mathrm{e}^{\mathrm{i}(\alpha x+\beta z)}$ and $\hat{\boldsymbol{f}}(y, t ; \alpha, \beta) \mathrm{e}^{\mathrm{i}(\alpha x+\beta z)}$ of streamwise and spanwise wavenumbers $\alpha$ and $\beta$ for which the following system is obtained (Pujals et al. 2009; Hwang \& Cossu 2010) from (2.1):

$$
\frac{\partial \hat{\boldsymbol{q}}}{\partial t}=\boldsymbol{A} \hat{\boldsymbol{q}}+\boldsymbol{B} \hat{\boldsymbol{f}}
$$

The state vector is formed with the wall-normal components of the velocity and vorticity Fourier modes $\hat{\boldsymbol{q}}=\left[\hat{v}, \hat{\omega}_{y}\right]^{\mathrm{T}}$. The operators $\boldsymbol{A}$ and $\boldsymbol{B}$ are defined as

$$
\boldsymbol{A}=\left[\begin{array}{cc}
\Delta^{-1} \mathscr{L}_{O \mathscr{S}} & 0 \\
-\mathrm{i} \beta U^{\prime} & \mathscr{L}_{\mathscr{S} \mathscr{Q}}
\end{array}\right], \quad \boldsymbol{B}=\left[\begin{array}{ccc}
-\mathrm{i} \alpha \Delta^{-1} \mathscr{D} & -k^{2} \Delta^{-1} & -\mathrm{i} \beta \Delta^{-1} \mathscr{D} \\
\mathrm{i} \beta & 0 & -\mathrm{i} \alpha
\end{array}\right],
$$

with the generalized Orr-Sommerfeld and Squire operators:

$$
\begin{aligned}
& \mathscr{L}_{O \mathscr{S}}=-\mathrm{i} \alpha\left(U \Delta-U^{\prime \prime}\right)+v_{T} \Delta^{2}+2 v_{T}^{\prime} \Delta \mathscr{D}+v_{T}^{\prime \prime}\left(\mathscr{D}^{2}+k^{2}\right), \\
& \mathscr{L}_{\mathscr{S} \mathscr{Q}}=-\mathrm{i} \alpha U+v_{T} \Delta+v_{T}^{\prime} \mathscr{D} .
\end{aligned}
$$

Here, $\Delta=\mathscr{D}^{2}-k^{2}, k^{2}=\alpha^{2}+\beta^{2}, \mathscr{D}$ and ' denote $\partial / \partial y$. Homogeneous boundary conditions are enforced on both walls: $\hat{v}( \pm h)=\mathscr{D} \hat{v}( \pm h)=\hat{\omega}_{y}( \pm h)=0$. Otherwise, when it is not specified, we will assume that homogeneous initial conditions $\hat{\boldsymbol{u}}_{0}=\mathbf{0}$ are used for the system. The velocity can be obtained from the state vector with

$$
\hat{\boldsymbol{u}}=\boldsymbol{C} \hat{\boldsymbol{q}}, \quad \boldsymbol{C}=\frac{1}{k^{2}}\left[\begin{array}{cc}
\mathrm{i} \alpha \mathscr{D} & -\mathrm{i} \beta \\
k^{2} & 0 \\
\mathrm{i} \beta \mathscr{D} & \mathrm{i} \alpha
\end{array}\right],
$$

and vice versa

$$
\hat{\boldsymbol{q}}=\boldsymbol{D} \hat{\boldsymbol{u}}, \quad \boldsymbol{D}=\left[\begin{array}{ccc}
0 & 1 & 0 \\
\mathrm{i} \beta & 0 & -\mathrm{i} \alpha
\end{array}\right] .
$$

The forcing term $f$ driving the system will be referred to as 'input' and the driven motions $\boldsymbol{u}$ as 'output'.

\subsection{Optimal harmonic and stochastic energy amplification}

When an harmonic forcing $\hat{\boldsymbol{f}}(y, t)=\tilde{\boldsymbol{f}}(y) \mathrm{e}^{\mathrm{i} \omega_{f} t}$ of real frequency $\omega_{f}$ drives the linearly stable system (2.3), for sufficiently large times, the response is $\hat{\boldsymbol{u}}(y, t)=\tilde{\boldsymbol{u}}(y) \mathrm{e}^{\mathrm{i} \omega_{f} t}$. Making use of (2.3) it is easily found that $\tilde{\boldsymbol{u}}=\boldsymbol{H} \tilde{\boldsymbol{f}}$, where the transfer function is 
$\boldsymbol{H}=\mathbf{C}\left(\mathrm{i} \omega_{f} \boldsymbol{I}-\boldsymbol{A}\right)^{-1} \boldsymbol{B}$. In this case, the optimal harmonic amplification is defined by optimizing the ratio of the energy of the output to the energy of the input and coincides with the square of the norm of the transfer function, i.e. the pseudospectrum evaluated on the imaginary axis (see e.g. Reddy et al. 1993; Trefethen et al. 1993)

$$
R\left(\omega_{f} ; \alpha, \beta\right)=\max _{\tilde{\boldsymbol{f}} \neq \mathbf{0}} \frac{\|\tilde{\boldsymbol{u}}\|^{2}}{\|\tilde{\boldsymbol{f}}\|^{2}}=\|\boldsymbol{H}\|^{2},
$$

where the standard energy norm is used, $\|\hat{\boldsymbol{u}}\|^{2}=\int_{-h}^{h} \hat{\boldsymbol{u}}^{H} \hat{\boldsymbol{u}} \mathrm{d} y$. The maximum response $R_{\max }(\alpha, \beta)=\max _{\omega_{f}} R\left(\omega_{f} ; \alpha, \beta\right)$, obtained with $\omega_{f, \max }$, is also referred to as the $H_{\infty}$ norm of the transfer function (Zhou, Doyle \& Glover 1996).

When a stochastic forcing drives the system (2.3), the response is also stochastic, and we are interested in its variance $V=\langle\|\hat{\boldsymbol{u}}\|\rangle$, where $\langle\cdot\rangle$ is the ensemble average. The variance can also be retrieved as the trace of the covariance tensor $\left\langle\hat{\boldsymbol{u}} \hat{\boldsymbol{u}}^{H}\right\rangle$. We follow previous investigations in considering a zero-mean $(\langle\hat{\boldsymbol{f}}\rangle=\mathbf{0})$ delta-correlated forcing with Gaussian probability density distribution which, for convenience, we also assume to be isotropic $\left\langle\hat{\boldsymbol{f}}(t) \hat{\boldsymbol{f}}^{H}\left(t^{\prime}\right)\right\rangle=\boldsymbol{I} \delta\left(t-t^{\prime}\right)$. In this case the variance can be obtained in the frequency or in the temporal domain as

$$
V(\alpha, \beta)=\frac{1}{2 \pi} \int_{-\infty}^{\infty} \operatorname{trace}\left(\boldsymbol{H} \boldsymbol{H}^{\dagger}\right) \mathrm{d} \omega=\operatorname{trace}\left(\mathbf{C} \boldsymbol{X}_{\infty} \mathbf{C}^{\dagger}\right),
$$

where the adjoints ${ }^{\dagger}$ are defined with respect to the inner product $(\hat{\boldsymbol{u}}, \hat{\boldsymbol{v}})=\int_{-h}^{h} \hat{\boldsymbol{u}}^{H} \hat{\boldsymbol{v}} \mathrm{d} y$. The variance represents the $H_{2}$ norm of the transfer function (Zhou et al. 1996). Following the temporal domain approach, one has to compute the solution $\boldsymbol{X}_{\infty}$ of the algebraic Lyapunov equation:

$$
\boldsymbol{A} \boldsymbol{X}_{\infty}+\boldsymbol{X}_{\infty} \boldsymbol{A}^{\dagger}+B \boldsymbol{B}^{\dagger}=0 .
$$

The covariance of the response is then retrieved as $\left\langle\hat{\boldsymbol{u}} \hat{\boldsymbol{u}}^{H}\right\rangle=\mathbf{C} \boldsymbol{X}_{\infty} \boldsymbol{C}^{\dagger}$. The covariance is self-adjoint and has real eigenvalues $\sigma_{j}$ with $V=\sum \sigma_{j}$, and a set of mutually orthogonal eigenfunctions often referred to as 'empirical orthogonal functions', Karhunen-Loève (KL) or 'proper orthogonal decomposition' (POD) modes. The ratio $\sigma_{j} / V$ represents the contribution of the $j$ th mode to the variance, and the corresponding eigenfunction provides the associated spatial structure in the response. The eigenfunction corresponding to the largest $\sigma_{j}$ is the optimal mode in the sense that it contributes most to the variance. The forcing profiles associated with each KL mode are obtained by solving the dual Lyapunov problem. For further details, the reader is referred to Farrell \& Ioannou (1993a,b), Zhou et al. (1996), Bamieh \& Dahleh (2001), Jovanović \& Bamieh (2005) and Schmid (2007).

The response to optimal harmonic forcing and the variance maintained by stochastic forcing are computed based on the generalized Orr-Sommerfeld-Squire system (2.3). A Chebyshev-collocation method is used to discretize the above equations in the wallnormal direction. The discretized differentiation operators of Weideman \& Reddy (2000), which include the appropriate boundary conditions, are used, and standard methods described in Schmid \& Henningson (2001), for example, are used to compute the optimal harmonic response. The stochastic response is obtained by solving the Lyapunov equation (2.6) with the lyap function in MATLAB. The codes for computing the optimal harmonic and stochastic responses have been validated in Hwang \& Cossu (2010). The results in this study are obtained by using from 129 to 513 collocation points as in Pujals et al. (2009). 

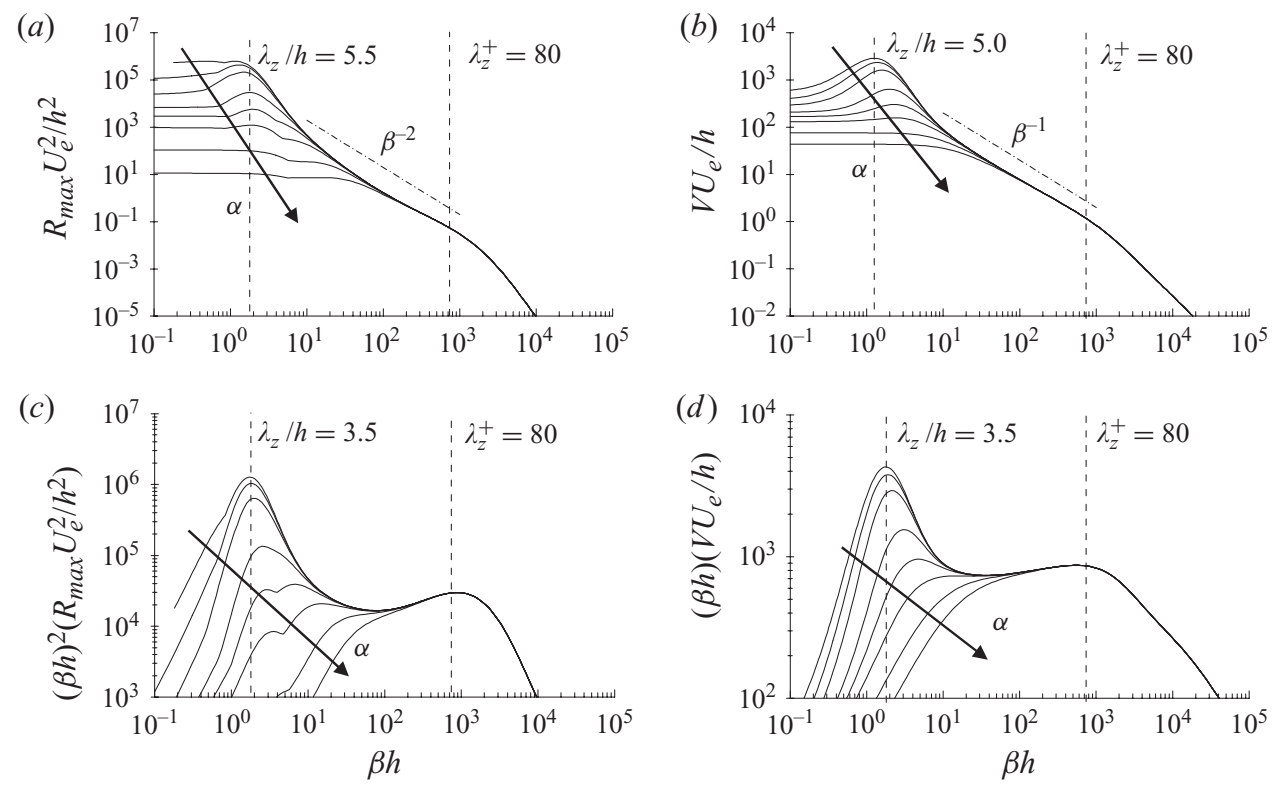

FIGURE 1. Energy amplifications and corresponding pre-multiplied energy amplifications of optimal harmonic and stochastic forcing at $R e_{\tau}=10000:(a) R_{\max }(\alpha, \beta),(b) V(\alpha, \beta)$, (c) $\beta^{2} R_{\max }(\alpha, \beta)$ and $(d) \beta V(\alpha, \beta)$. Here, $\alpha h=0.0,0.1,0.2,0.5,1.0,2.0,5.0,10.0$, outer to inner curves.

\section{Energy amplifications at $\boldsymbol{R e}_{\tau}=10000$}

\subsection{Wavenumber dependence of the energy amplifications}

We begin by considering the single Reynolds number $R e_{\tau}=10000 . R_{\max }$ and $V$ have been computed for a set of selected streamwise and spanwise wavenumbers and are reported in figures $1(a)$ and $1(b)$, respectively. In both cases, the largest amplifications are reached for streamwise uniform perturbations $(\alpha=0)$. Only streamwise-elongated perturbations $(\alpha \leqslant \beta)$ are significantly amplified. $R_{\max }$ and $V$ have a peak at $\beta=$ $1.25 / h\left(\lambda_{z}=5.0 h\right)$ and $\beta=1.14 / h\left(\lambda_{z}=5.5 h\right)$, respectively, and they monotonously decrease as $\beta$ increases, with a noticeable change of slope near $\lambda_{z}^{+} \approx 80$. For spanwise wavenumbers roughly in the range $15<\beta h<400$ (and for $\alpha \ll \beta$ ), however, both $R_{\max }$ and $V$ show an approximate linear dependence on $\beta$ in $\log -\log$ coordinates denoting a power-law dependence. A best fit in this range reveals a $\beta^{-2}$ scaling for $R_{\max }$ and a $\beta^{-1}$ scaling for $V$. A detailed discussion of these scalings is deferred to $\S 5$, where we will show that they are associated to geometrical similarity of optimal structures in the log layer. The deviations from the approximate $\beta^{-2}$ and $\beta^{-1}$ scalings are revealed by a pre-multiplication of $R_{\max }$ and $V$ with $\beta^{2}$ and $\beta$, respectively. The pre-multiplied amplification curves, reported in figures $1(c)$ and $1(d)$, reveal the same double-peaked structure already observed for the optimal transient growth by del Álamo \& Jiménez (2006) and Pujals et al. (2009). For both $R_{\max }$ and $V$, the peak associated with the streamwise uniform large-scale structures is found at $\beta h \simeq 1.8$ $\left(\lambda_{z} / h \simeq 3.5\right)$, while that associated with near-wall streaks is found at $\beta^{+} \simeq 0.079$ $\left(\lambda_{z}^{+} \simeq 80\right)$. These two optimal wavelengths delimit the range where the approximate power law can apply. From figures $1(c)$ and $1(d)$, it is also seen how, however, even at this very large $R e_{\tau}$, the influence of the inner and outer scales is also felt well inside the intermediate wavenumber range. 

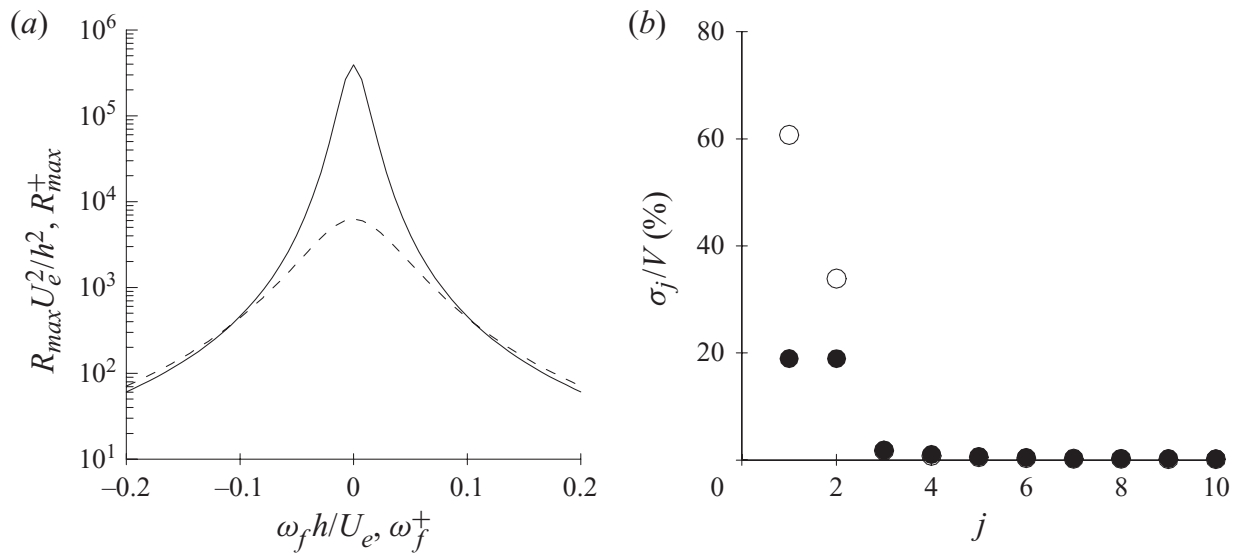

FIGURE 2. (a) Optimal harmonic amplification, $R$, plotted versus the forcing frequency, $\omega_{f}$, in correspondence to the outer peak at $\lambda_{z}=3.5 \mathrm{~h}$ and to the inner peak at $\lambda_{z}^{+}=80$. (b) Contributions $\sigma_{j} / V$ of the first $10 \mathrm{KL}$ modes to the variance of the stochastic response in correspondence to the outer peak at $\lambda_{z}=3.5 \mathrm{~h}(\mathrm{O})$ and to the inner peak at $\lambda_{z}^{+}=80(\mathbf{\bullet})$. Streamwise uniform perturbations $(\alpha=0)$ are considered.

\subsection{Optimal structures}

In figure 2, we analyse the 'selectivity' of the amplifications obtained in correspondence to the outer and inner peaks of the pre-multiplied amplifications for $\lambda_{x}=\infty(\alpha=0)$, $\lambda_{z}=3.5 h$ and $\lambda_{z}^{+}=80$. For the structures associated to both the inner and the outer peak, the system behaves like a low-pass frequency filter, as seen from figure $2(a)$, where the dependence of the optimal response $R$ on the forcing frequency $\omega_{f}$ is reported. The maximum amplification is reached with steady forcing $\left(\omega_{f, \max }=0\right)$; the corresponding optimal structures are reported in figures 3(a) and 3(c), respectively. As expected, the optimal forcing represents streamwise vortices, while the optimal responses are streamwise streaks. The structures associated with the near-wall peak are located in the buffer layer, where they have a maximum amplitude at $y^{+} \approx 10$, as for the experimentally observed near-wall streaks. The optimal forcing associated with the outer peak extends to the whole channel width and induces alternated highand low-speed streamwise streaks on both walls.

The most energetic KL (POD) modes have been computed to seek the structures with the largest contribution to the variance $V$ of the stochastic response in correspondence to the inner and outer peaks. From figure 2(b), where the contributions of the first $10 \mathrm{KL}$ modes are reported, we see that for the outer peak, the dominant mode contributes to $64 \%$ of the total variance. This mode fills the whole wall-normal domain (see figure $3 b$ ). For the wavenumbers associated to the inner peak, two dominant modes are found, each contributing to $23 \%$ of the total variance. These two modes are associated to identical near-wall structures, respectively, located on the upper and the lower walls (see also figure $3 d$ ). Each mode can be obtained by the other one via a $y \rightarrow-y$ transformation.

The optimal perturbations reported in figure 3, corresponding to harmonic and stochastic forcing, respectively, are very similar. We further examine this resemblance by comparing in figure 4 their optimal inputs and outputs for wall-normal profiles. We also include, for comparison, the optimal perturbations computed by Pujals et al. (2009) for the initial-value problem. The optimal inputs and outputs of the three 

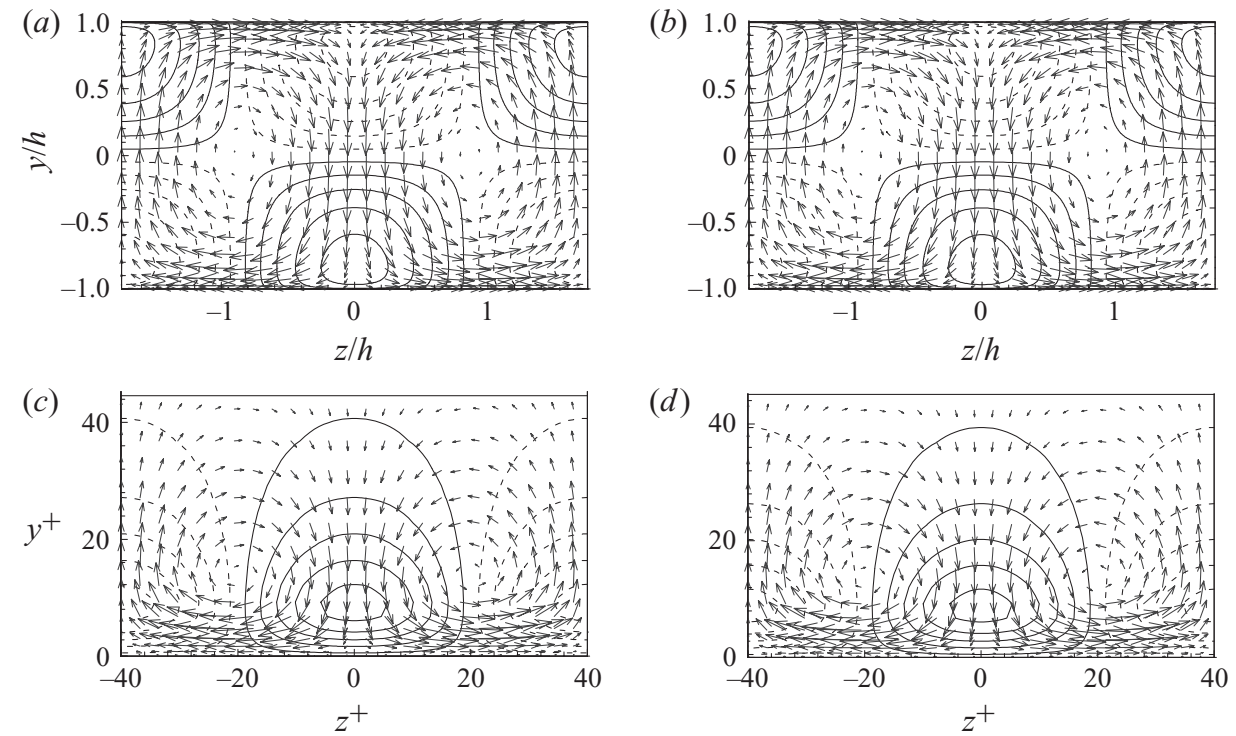

FiguRE 3. Cross-stream $(y-z$ plane $)$ view of the normalized optimal forcing and the corresponding response for spanwise wavenumbers corresponding to $(a, b)$ the outer peak $\left(\lambda_{z}=3.5 h\right)$ and $(c, d)$ inner peak $\left(\lambda_{z}^{+}=80\right)$ for harmonic forcing $(a, c)$ and stochastic forcing $(b, d)$. The solid and dashed contours denote the positive and negative streamwise velocity of the responses with increment of 0.2 from -0.9 to 0.9 , respectively, and the vectors represent the cross-streamwise fields of the forcing. The perturbations are streamwise uniform $(\alpha=0)$.

different problems are almost identical. The optimal wall-normal input components (vortices) associated to the outer peaks have their maxima at the channel centre (figure $4 a$ ), and those associated to the inner peaks have their maxima at $y^{+} \simeq 15$ (figure $4 c$ ). The maxima of the output streaks for the outer peaks are located at $|y / h|=0.8$ (i.e. at a distance of $0.2 h$ from the walls, figures $4 b$ and $4 d$ ), while those for the inner peaks reach a maximum amplitude at $y^{+} \simeq 10$ (figures $4 b$ and $4 d$ ).

The resemblance of the optimal harmonic and stochastic forcing optimals is also observed for spanwise wavenumbers situated in the intermediate range between the two peaks of the pre-multiplied amplification curves reported in figures $4(b)$ and $4(e)$. The wall-normal maxima of these optimals are situated in the log layer (between $y^{+} \approx 50$ and $y \approx 0.2 h$ ) and their amplitude is negligible in the outer layer.

For the initial-value problem, del Álamo \& Jiménez (2006) have found that, in the intermediate range, the optimal structures are, to a first approximation, geometrically similar. We have therefore plotted their profiles in the similarity variable $y^{+} / \lambda_{z}^{+}=(y+h) / \lambda_{z}$ for three representative wavenumbers in the intermediate range. From figures $4(b)$ and $4(e)$, we see that the optimal input and output for the stochastic and harmonic forcing are, to a first approximation, geometrically similar. Their rescaled shape is also not very different from that of the buffer-layer structures if those ones are re-scaled on their wavelength $\lambda_{z}^{+}=80$.

The results found for streamwise uniform structures $(\alpha=0)$ extend to the $\alpha \ll \beta$ case with only minor modifications. When $\alpha \neq 0$, the optimal forcing frequency is different from zero in the harmonic forcing case, and the real and imaginary part of the optimal perturbations display a slight tilting in the streamwise direction reminiscent of the Orr effect for purely two-dimensional perturbations (see e.g. Butler \& Farrell 1992; 

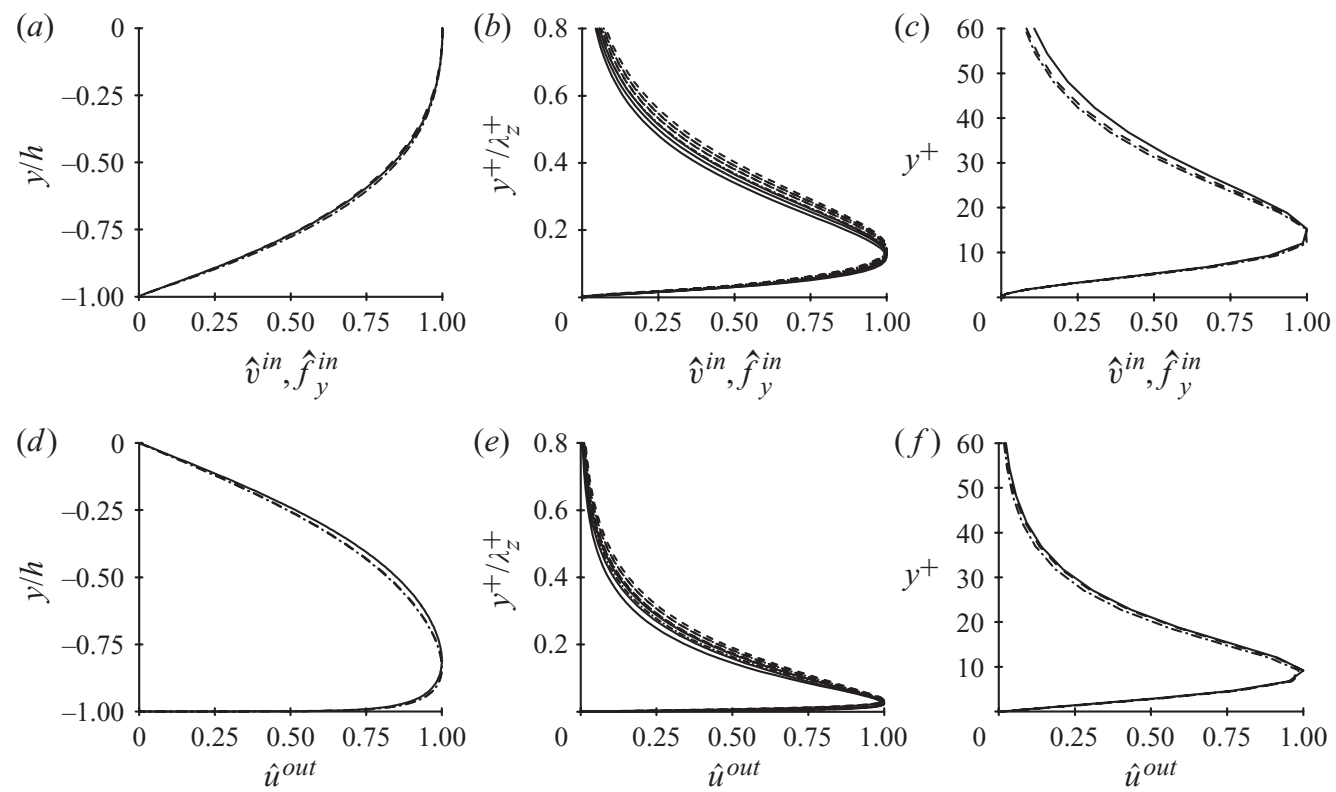

Figure 4. Profiles of streamwise uniform $(\alpha=0)$ optimal modes corresponding to the outer peak $(a, d)$, the intermediate wavenumber range $(b, e)$ and the inner peak $(c, f)$ of the pre-multiplied amplifications. $(a-c)$ Wall-normal components of the input (initial condition $\hat{v}^{i n}(y)$ for transient growth and forcing $\hat{f}_{y}^{\text {in }}(y)$ for harmonic and stochastic forcing); $(d-f)$ streamwise components of the corresponding outputs $\hat{u}^{\text {out }}(y)$. Here, - , optimal transient growth; - - - , optimal harmonic forcing; ----- , stochastic excitation. The outer and inner peaks correspond, respectively, to $\lambda_{z}=4 h$ and $\lambda_{z}^{+}=92$ for transient growth (see also Pujals et al. 2009), $\lambda_{z}=3.5 \mathrm{~h}$ and $\lambda_{z}^{+}=80$ for harmonic and stochastic forcing. In the intermediate $\beta$ range between the outer and inner peaks, the wavenumbers $\beta^{+}=0.01 / h, 0.005 / h, 0.003 / h$ $\left(\lambda_{z}^{+}=628,1257,2094\right)$ are chosen. In $(b, d)$, the profiles are plotted versus $y / \lambda_{z}$ to account for the geometrical similarity of log-layer structures.

Schmid \& Henningson 2001). However, the wall-normal amplitude of the optimal perturbations changes only slightly with respect to the streamwise uniform case, as shown in figure 5 where wavelengths typical of experimental observations $\left(\lambda_{z}=3.5 \mathrm{~h}\right.$, $\left.\lambda_{x}=20 h\right)$ and $\left(\lambda_{z}^{+}=100, \lambda_{x}^{+}=600\right)$ are considered. Also in this case, the optimal inputs and outputs obtained with the three different analyses (initial value, harmonic forcing and stochastic forcing) are almost indistinguishable (not shown).

\section{Dependence on the Reynolds number}

The optimal amplifications have been computed for selected values of $R e_{\tau}$ ranging from 500 to 20000 . For the $R e_{\tau}$ considered, it is found that the streamwise uniform $(\alpha=0)$ perturbations are the most amplified ones (not shown). The respective inner and outer scaling of the inner and outer peaks for both the response to harmonic and to stochastic forcing is clearly seen in figure 6, where the pre-multiplied optimal amplifications for $\alpha=0$ are reported versus the spanwise wavenumber $\beta$ scaled in outer and inner units. The maximum amplifications associated with the outer peak clearly increases with $R e_{\tau}$, as already found by Pujals et al. (2009) and Cossu, Pujals \& Depardon (2009) in the optimal transient growth case. The amplifications 

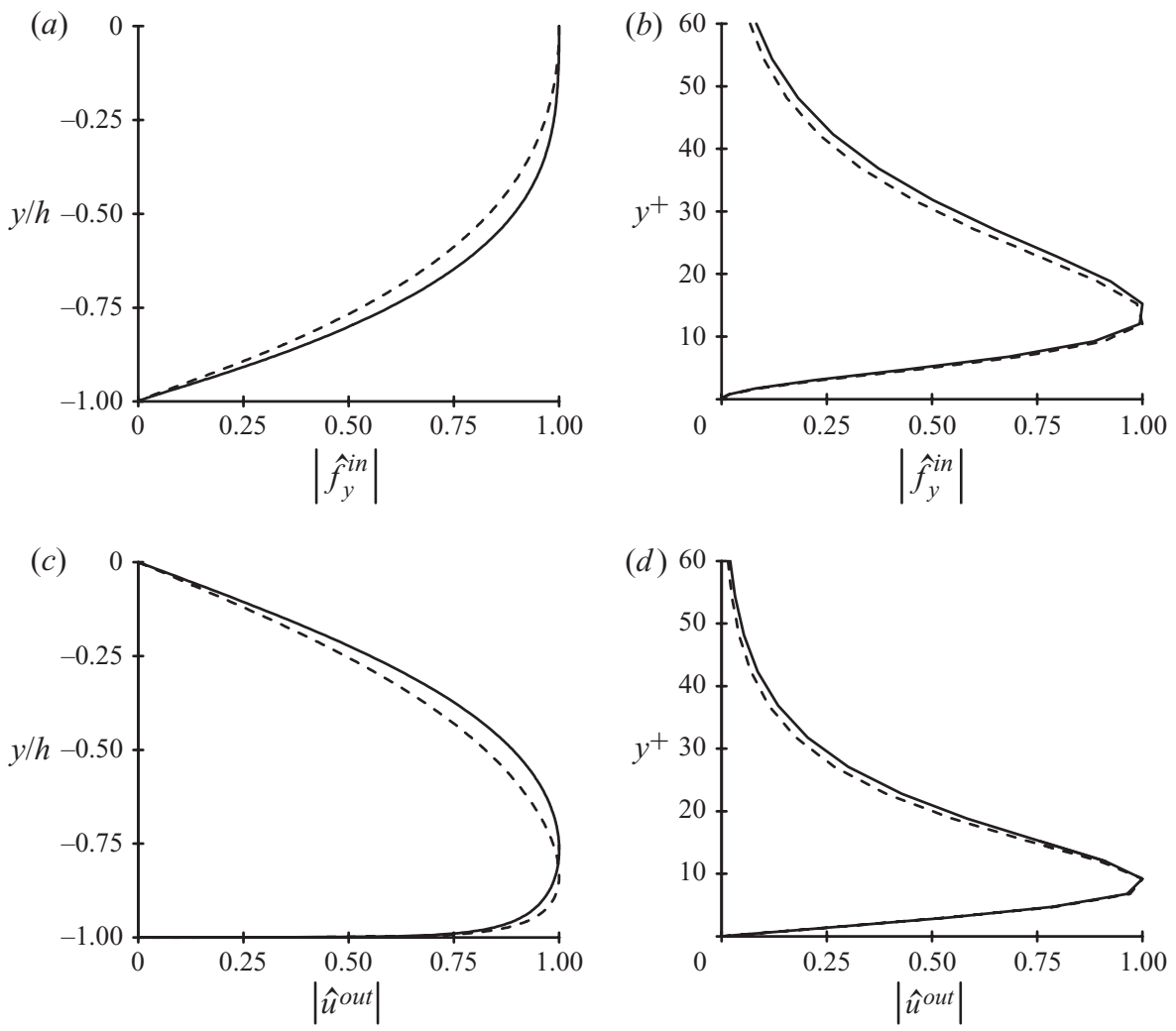

FiguRE 5. Solid lines: profiles of optimal modes with $\lambda_{x} \approx 6 \lambda_{z}(\alpha=0.167 \beta)$ for $(a, c)$ wavelengths in the neighbourhood of the outer peak $\left(\lambda_{z}=3.5 h, \lambda_{x}=20 h\right)$ and $(b, d)$ wavelengths in the neighbourhood of the inner peak $\left(\lambda_{z}^{+}=100, \lambda_{x}^{+}=600\right)$. $(a, b)$ Wall-normal components of the optimal input $\left|\hat{f}_{y}^{\text {in }}\right|(y)$ amplitude for harmonic forcing; $(c, d)$ streamwise components of the amplitude of the corresponding output $|\hat{u}|(y)$. The streamwise uniform perturbations $\left(\alpha=0, \lambda_{x}=\infty\right)$ with the same spanwise wavelength are also reported for comparison as dashed lines. Only the perturbations for the optimal harmonic forcing are reported; those for the optimal initial-value problem and for stochastic forcing are almost indistinguishable.

associated with the inner peak do not sensibly change with $R e_{\tau}$, when re-scaled in inner units.

The shape of the optimal input and output harmonic forcing profiles for, respectively, the inner and outer peaks already reported in figure 4 are re-plotted in figure 7 for the considered Reynolds numbers $R e_{\tau}$ ranging from 500 to 20000 . From this figure, it is apparent that their shape does not change with the Reynolds number when it is plotted in, respectively, inner and outer units. This extends to the harmonic forcing case the results for the initial-value problem found by Pujals et al. (2009) for the turbulent channel and by Cossu et al. (2009) in the turbulent boundary layer. Exactly the same results are found when considering the maximum energy KL (POD) modes for the stochastic forcing case. Pujals et al. (2009) and Cossu et al. (2009) also observed that when the wall-normal profile $\hat{u}(y)$ of the optimal streaks corresponding to the outer peak are scaled in inner units, they coincide in the inner layer $(y<\approx 0.1 h)$, where they are proportional to the mean-flow profile $U_{b}(y)$. Here, we find that the same applies to the optimal responses to forcing, as can be seen 

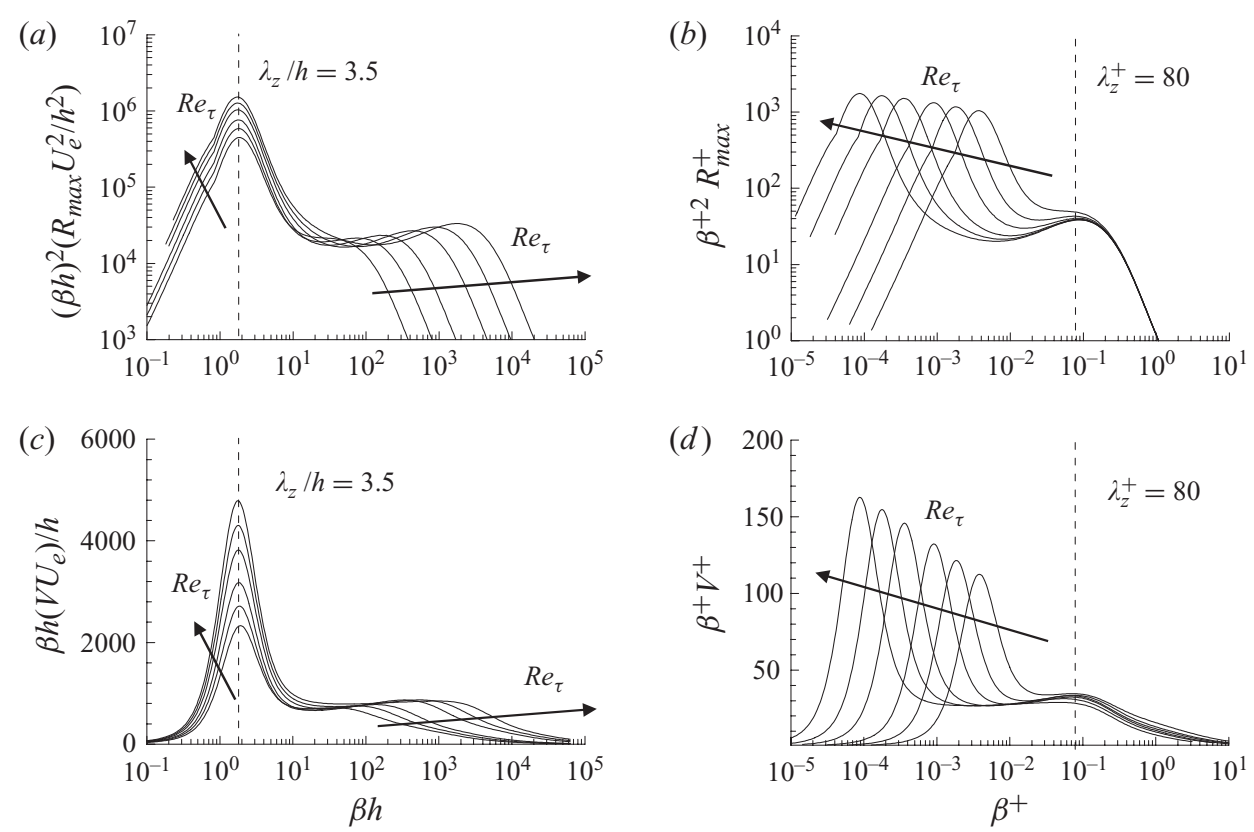

FIGURE 6. Pre-multiplied responses to $(a, b)$ optimal harmonic forcing and $(c, d)$ stochastic excitation with respect to the spanwise wavenumber for the Reynolds numbers $R e_{\tau}=500,1000,2000,5000,10000$ and 20000: $(a, c)$ scaling in the outer units; $(b, d)$ scaling in the inner units.

(a)

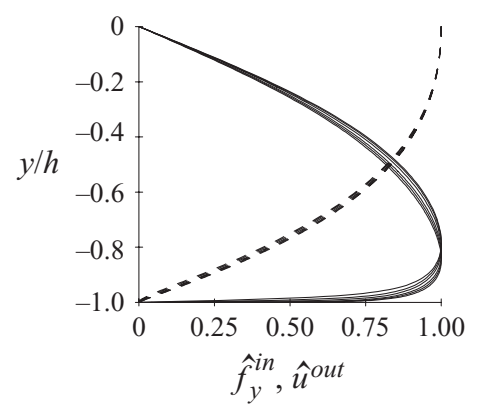

(b)

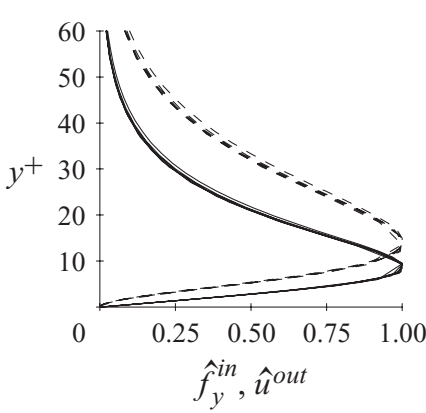

(c)

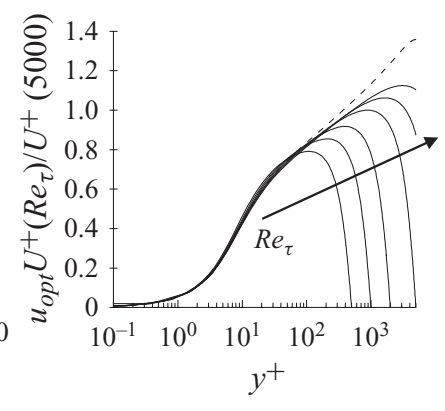

FIGURE 7. Profiles of the wall-normal component $\hat{f}_{y}^{i n}(y)$ of the optimal harmonic forcing and the streamwise component $\hat{u}^{\text {out }}(y)$ of its response at $(a)$ the outer peak $(\alpha h=0, \beta h=1.8)$ and (b) the inner peak $\left(\alpha^{+}=0, \beta^{+}=0.079\right)$ for $R e_{\tau}=500,1000,2000,5000,10000$ and 20000 . Note that all the curves are almost indistinguishable. Here,,$---- \hat{f}_{y}^{\text {in }} ;-, \hat{u}^{\text {out }}$. (c) Outer peak streamwise velocity response $\hat{u}^{\text {out }}(y)$ scaled with a factor $U_{e}^{+}\left(R e_{\tau}\right) / U_{e}^{+}(5000)$ for the selected Reynolds numbers. The re-scaled mean flow profile at $R e_{\tau}=5000$ (dashed line) is also shown for comparison.

in figure $7(c)$ where the optimal outer-peak streak amplitudes $\hat{u}$ are plotted in inner units. From figure 7(c), it is also seen that the optimal streaks associated to the outer peak deeply penetrate into the buffer layer; as in the optimal transient growth case, the streaks at $y^{+}=12$ still exhibit half of their maximum amplitude. 


\section{Scaling of optimal amplifications of geometrically similar log-layer structures}

The numerical results reported in $\S 3$ have shown that the amplifications $R_{\max }$ and $V$ of the most amplified streamwise-elongated perturbations $(\alpha \ll \beta)$ exhibit an approximate $\beta^{-2}$ and $\beta^{-1}$ scaling, respectively, in the intermediate $\beta$ range. The data reported in figures $4(b)$ and $4(e)$ also show that the associated optimal input and outputs are, to first order, geometrically similar, consistent with the previous findings of del Álamo \& Jiménez (2006). Furthermore, these optimal perturbations have maximum amplitude in the log layer, and have negligible amplitudes in the outer layer. In $\S 5$, we show that the observed $\beta^{-2}$ and $\beta^{-1}$ scalings can be directly deduced from the generalized Orr-Sommerfeld-Squire model (2.3) under the assumptions of log-layer behaviour of the base-flow profile and of geometric similarity of the perturbations. We consider streamwise uniform $(\alpha=0)$ perturbations that, besides being the most amplified ones, are also easily tractable.

Spanwise periodic, geometrically similar streamwise uniform velocity and forcing fields are identical in the variables $y^{+} / \lambda_{z}^{+}=(y+h) / \lambda_{z}, z^{+} / \lambda_{z}^{+}=z / \lambda_{z}$. As a consequence, their Fourier amplitudes at different $\lambda_{z}$ are given by the same function of the re-scaled wall-normal coordinate $\check{y}=\bar{\beta}(y+h)$ with $\bar{\beta}=(\beta / \check{\beta})$ so that $(y+h) / \lambda_{z}=\check{y} / \check{\lambda}_{z}$, where the reference spanwise wavenumber $\check{\beta}$ can be arbitrarily chosen. The re-scaled state vector is defined as $\check{\boldsymbol{q}}=\left[\hat{v}, \hat{\omega}_{y} / \bar{\beta}\right]^{\mathrm{T}}(\check{y}, t)$. The re-scaling of the wall-normal vorticity is due to the fact that, for streamwise uniform perturbations, $\hat{\omega}_{y}=\mathrm{i} \beta \hat{u}$, and therefore the similarity is obtained on $\hat{\omega}_{y} / \beta$ instead of $\hat{\omega}_{y}$. In the Appendix we detail how, assuming a logarithmic behaviour for the base flow and reformulating system (2.3) in terms of re-scaled variables, the following system is obtained: $\partial \check{\boldsymbol{q}} / \partial t=\bar{\beta} \check{\boldsymbol{A}} \check{\boldsymbol{q}}+\check{\boldsymbol{B}} \hat{\boldsymbol{f}}$, with $\hat{\boldsymbol{u}}=\check{\boldsymbol{C}} \check{\boldsymbol{q}}$ and for the initial conditions $\check{\boldsymbol{q}}_{0}=\check{\boldsymbol{D}} \hat{\boldsymbol{u}}_{0}$. The operators $\check{\boldsymbol{A}}, \check{\boldsymbol{B}}, \check{\boldsymbol{C}}$ and $\check{\boldsymbol{D}}$ are obtained by simply evaluating $\boldsymbol{A}, \boldsymbol{B}, \boldsymbol{C}$ and $\boldsymbol{D}$ at the reference $\check{\beta}$ and $\alpha=0$. These operators therefore do not depend on $\beta$.

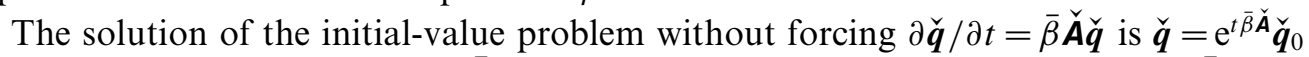
and depends on time only via $\bar{\beta} t$. We therefore define the re-scaled time $\bar{t}=\bar{\beta} t$ and rewrite the system as

$$
\frac{\partial \check{\boldsymbol{q}}}{\partial \bar{t}}=\check{\boldsymbol{A}} \check{\boldsymbol{q}}+\bar{\beta}^{-1} \check{\boldsymbol{B}} \hat{\boldsymbol{f}} .
$$

In the case of the initial-value problem without forcing, the solution in terms

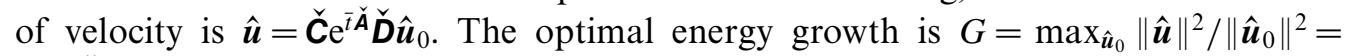

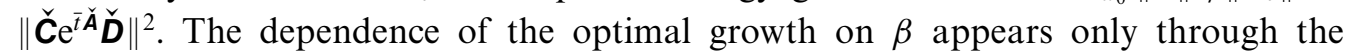
re-scaled time and therefore

$$
G(\beta, t)=G(\check{\beta}, \bar{t} \check{\beta} / \beta) .
$$

The optimal growth for a given $\beta$ is given by the optimal growth computed at the reference $\breve{\beta}$ re-scaled in time with a factor $\beta / \beta$. The time at which the maximum growth is attained, $t_{\max }=\bar{t}_{\max } \check{\beta} / \beta$, is therefore proportional to the wavelength $\lambda_{z}$ of the perturbation, while the maximum growth $G_{\max }=\max _{t} G(t)$ does not depend on $\beta$. These properties have indeed been observed in the computations of optimal transient growth (del Álamo \& Jiménez 2006; Cossu et al. 2009; Pujals et al. 2009) for the range of spanwise wavenumbers associated with log-layer structures.

In the case of harmonic forcing, the re-scaled forcing frequency $\bar{\omega}_{f}=\omega_{f} / \bar{\beta}$ can be defined such that $\omega_{f} t=\bar{\omega}_{f} \bar{t}$. From (5.1) it then follows that the harmonic response $\hat{\boldsymbol{u}}=\tilde{\boldsymbol{u}} \mathrm{e}^{\mathrm{i} \bar{\omega}_{f} \bar{t}}$ to the harmonic forcing $\hat{\boldsymbol{f}}=\tilde{\boldsymbol{f}} \mathrm{e}^{\mathrm{i} \bar{\omega}_{f} \bar{t}}$ is $\tilde{\boldsymbol{u}}=\overline{\boldsymbol{\beta}}^{-1} \check{\boldsymbol{H}} \tilde{\boldsymbol{f}}$, where 
$\check{\boldsymbol{H}}=\check{\boldsymbol{C}}\left(\mathbf{i} \bar{\omega}_{f} \boldsymbol{I}-\check{\boldsymbol{A}}\right)^{-1} \check{\boldsymbol{B}}$. The optimal amplification is $R=\bar{\beta}^{-2}\|\check{\boldsymbol{H}}\|^{2}$ which implies that

$$
R\left(\beta, \omega_{f}\right)=\left(\frac{\check{\beta}}{\beta}\right)^{2} R\left(\check{\beta}, \bar{\omega}_{f} \beta / \check{\beta}\right) .
$$

The maximum optimal harmonic amplification $R_{\max }$ therefore scales like $\beta^{-2}$ and is found for $\omega_{f, \max }=\bar{\omega}_{f, \max } \beta / \check{\beta}$. These predictions agree with what is observed, to a first approximation, for the optimal harmonic amplification in the intermediate wavenumber regime.

Finally, the variance $V$ of the response to stochastic forcing can be obtained in the frequency domain as $V=(1 / 2 \pi) \int_{-\infty}^{\infty} \operatorname{trace}\left(\boldsymbol{H} \boldsymbol{H}^{\dagger}\right) \mathrm{d} \omega$. Under the assumptions of $\S 5$, $\boldsymbol{H}=\bar{\beta}^{-1} \check{\boldsymbol{H}}$ so that, changing the integration variable to $\omega=\bar{\beta} \bar{\omega}$, it is found that $V=\left(\bar{\beta}^{-1} / 2 \pi\right) \int_{-\infty}^{\infty} \operatorname{trace}\left[\left(\check{H}^{\dagger} \dot{H}^{\dagger}\right) \mathrm{d} \bar{\omega}\right.$ and therefore

$$
V(\beta)=\left(\frac{\check{\beta}}{\beta}\right) V(\check{\beta}) .
$$

The variance therefore scales like $\beta^{-1}$, as found from the numerical results reported in $\S 3$.

\section{Discussion}

6.1. Remarks on the scaling of geometrically similar log-layer perturbations

The numerical results reported in $\S 3$ have shown that in an intermediate $\beta$ range, the optimal perturbations exhibit an approximate geometrical similarity in the log layer, which is completely compatible with the concept of geometrically similar 'attached' eddies proposed by Townsend (1976) and further developed in the theory of Perry \& Chong (1982) and many others, and with the experimental findings of Morrison \& Kronauer (1969).

In the intermediate $\beta$ range, where the approximate geometrical similarity is observed, the amplification of the optimal harmonic and stochastic forcing displays an approximate $\beta^{-2}$ and $\beta^{-1}$ scaling. Exactly the same $\beta$ scalings are predicted by assuming geometrically similar perturbations and a log-layer form for the base flow and the associated eddy viscosity in the generalized Orr-Sommefeld-Squire operators, as shown in $\S 5$. The examination of the pre-multiplied amplification curves, however, reveals that the power-law dependence is only a first-order approximation and that the influence of the outer and inner scales is important even in the intermediate wavenumber regime, where a sort of plateau would be expected. A possible explanation for this, among others, is that the considered Reynolds numbers are not sufficiently high for a well-defined plateau to exist. The examination of figure 6 reveals that, actually, a sort of plateau seems to develop when the Reynolds number is increased, even if no definite conclusion can be drawn from these results. However, an order of magnitude of the minimum Reynolds number necessary to observe the hypothetical plateau, can be evaluated from the existing data. From figure $4(e)$ it is seen that in the geometrically similar regime the optimal response (streaks) amplitude $\hat{u}(y)$ becomes negligible for $y^{+}>\approx 0.6 \lambda_{z}^{+}$, while it is maximum at $y^{+} \approx 0.05 \lambda_{z}^{+}$. Let us assume that the log layer extends from $y^{+} \approx 50$ to $(y+h) \approx 0.2 h$. If the maximum of the geometrically similar streaks has to be in the log layer then $0.05 \lambda_{z}^{+}>50$, i.e. $\lambda_{z}^{+}>\lambda_{z, \min }^{+}=1000$ and in outer units $\lambda_{z, \min }=1000 h / R e_{\tau}$. If it is further required that the streak amplitude is negligible outside the log layer 
then $0.6 \lambda_{z}<0.2 h$, i.e. $\lambda_{z}<\lambda_{z, \max }=h / 3$. The condition $\lambda_{z, \max } / \lambda_{z, \text { min }}>1$ leads to the requirement $R e_{\tau}>O\left(10^{3}\right)$. If it is required that a plateau exist on at least two wavelength (or wavenumber) decades then the condition is $R e_{\tau}>O\left(10^{5}\right)$, which suggests that the considered Reynolds numbers may not be sufficiently high to observe a well-defined plateau in the pre-multiplied amplification curves. However, other possible explanations for the absence of a well-defined plateau may exist, such as a structural lack of exact similarity of the log-layer structures even at large $R e_{\tau}$. This issue therefore has to be considered unsolved.

\subsection{Non-normality and the large influence of large-scale optimal perturbations on the near-wall velocity field}

A noteworthy feature of the optimal streaks at large scale is that, even if they scale in outer units, they strongly penetrate into the inner layers where they still posses a large fraction of their maximum amplitude (as seen e.g. in figures $4 d, 5 c, 7 a$ and $7 c$ ). These optimal output streaks are induced by optimal inputs (initial condition or forcing) that are located essentially in the outer layer (see e.g. figures $4 a, 5 a$ and $7 a$ ). The forcing of optimal input vortices in the outer layer therefore leads to a large response in the inner layer. This is the case even when the input $\boldsymbol{g}$ is not optimal. The input can, indeed, be decomposed in a part parallel to the optimal input and another part orthogonal to it as $\boldsymbol{g}=A \boldsymbol{f}^{(\text {opt) }}+\boldsymbol{g}^{\perp}$ (where parallel and orthogonal are meant in the sense of the inner product used to define the energy norm, $f^{(o p t)}$ is normalized to unit energy and $A$ is the amplitude of the projection of $g$ on $f^{(o p t)}$ ). As the system is linear, the response to $g$ is the sum of the responses to $A f^{(o p t)}$ and $\mathbf{g}^{\perp}$, respectively. As the response to $\boldsymbol{f}^{(\text {opt })}$ is very large, the global output streak will have a large component proportional to the optimal large-scale streak, except in the very special case where $A$ is negligible. As, furthermore, the maximum amplification associated to the peak scaling in outer units increases with the Reynolds number $R e_{\tau}$, the influence of the outer-layer motions on the inner-layer streamwise velocity is predicted to increase with $R e_{\tau}$. This is what is observed in the real turbulent flows.

The fact that outer-layer motions can influence the near-wall region has been known at least since the seminal study of Townsend (1976). This influence has usually been interpreted 'kinematically' in terms of the velocity field directly induced by the outer motions. What is revealed by this study, and the previous ones in the same spirit, is that the large influence of outer-layer motions on the near-wall streamwise velocity is mainly 'dynamic', and based on the strong non-normality of the underlying linearized operator. The increase of the outer-layer influence with the Reynolds number is also naturally embedded in this non-normal dynamic mechanism. This interpretation also predicts that, when an integral over all spanwise wavelengths is performed, the total streamwise variance near the wall does not scale only in inner units, when components from motions at large scale are considered, and that an inner units scaling will be less and less accurate when $R e_{\tau}$ is increased. These qualitative predictions are compatible with the observations of De Graaff \& Eaton (2000), Hunt \& Morrison (2000), Metzger \& Klewicki (2001), del Álamo et al. (2004) and Hutchins \& Marusic (2007a). However, note that recent studies reveal that large-scale outer motions are also able to modulate the amplitude of the near-wall cycle (see, e.g. Hutchins \& Marusic 2007b; Mathis et al. 2009). This effect, which is probably nonlinear, is not taken into account by the present linear analysis. 
(a)

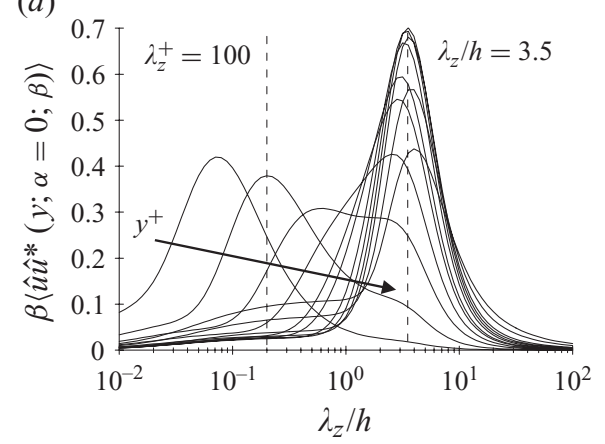

(b)

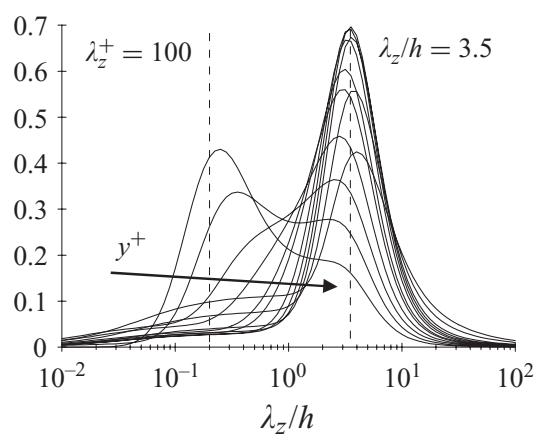

Figure 8. Pre-multiplied spectral density at each wall-normal location for the streamwise uniform case $\beta \hat{u} \hat{u}^{*}(y ; \alpha=0, \beta)$ based on the response to stochastic forcing $(a)$ without wall-damping function and $(b)$ with wall-damping function $f_{d}(y)=1-\exp \left(-\left(y^{+} / A^{+}\right)^{3}\right)$, where $A^{+}=25$. Here, the wall-normal location is chosen to be $y^{+}=5,15,29,48,71,98,155$, $202,309,402,500$ and each pre-multiplied spectral density is normalized by its area to emphasize its contents in spanwise wavenumbers. The considered Reynolds number is $R e_{\tau}=500$ to allow comparison with Jiménez (1998).

\subsection{Relevance of the stochastic forcing analysis}

When trying to relate the results of the present analysis to experimental observations of unforced turbulent flows, considering stochastic forcing is probably more relevant than considering harmonic forcing. In the spirit of Farrell \& Ioannou (1994) and Farrell \& Ioannou (1998), the stochastic forcing can be assumed to be a surrogate for the nonlinear terms that are neglected in the linearized model. The distribution $V(\alpha, \beta)$ would then represent the linear amplification of the variance of the nonlinear terms, where the whole variance integrated in the wall-normal direction is considered. More detailed information can, however, be extracted from the operator $\mathbf{C} \boldsymbol{X}_{\infty} \boldsymbol{C}^{\dagger}$, which gives the spatial spectral density amplification tensor of the correlations of $\hat{u}_{i}^{*}(y, t) \hat{u}_{j}\left(y^{\prime}, t\right)$. This tensor has been computed assuming that the forcing variance is uniform in $y$, which is not a realistic or even qualitatively correct assumption, especially near the wall. This can be seen in figure $8(a)$, where we report the normalized spanwise pre-multiplied spectra $\beta E_{\text {uи }}(\alpha=0, \beta)$ at selected distances $y$ from the wall for $R e_{\tau}=500$. The agreement of these curves with those reported by Jiménez (1998) and based on DNS data (at $R e_{\tau}=590$ ) is obviously only qualitative: the outer-layer spanwise peak predicted at $\lambda_{z} \approx 3.5 \mathrm{~h}$ is found at $1.5-2 h$ in the DNS. Furthermore, at $y^{+}=5$ the peak is found at values well below the expected $\lambda^{+} \approx 100$. Upgrading the simple Gaussian probability distribution to something more refined would probably not have a large effect on the presented results, as only low-order statistics are considered. However, a better modelling of the wall-normal distribution of the forcing can probably improve the results. For instance, it is not likely that the nonlinear terms at $y^{+}=5$ have the same amplitude as, for instance, in the buffer layer, where the near-wall self-sustaining cycle is located. If, then, the operator $\mathbf{C} \boldsymbol{X}_{\infty} \boldsymbol{C}^{\dagger}$ is recomputed, enforcing that the variance of the forcing decreases in the viscous sublayer, then the pre-multiplied amplification spectra at $y^{+}=5$ are improved, as shown in figure $8(b)$.

More realistic forcing distributions have not been considered, on purpose. Actually, the most relevant result of the presented stochastic response analysis is that, even with this extremely unrealistic input (Gaussian isotropic flat spectrum, uniform in 
the wall-normal direction), the output displays properties observed in real turbulent flows (strongly anisotropic velocity field with a dominant streak component, strongly non-flat spectrum with spanwise scales in the range of those observed in the real flows, approximate geometric similarity for intermediate wavenumbers). The fact that qualitatively 'realistic' spanwise scales are selected by the non-normal linear amplification mechanism suggests that this mechanism plays an important role in the real turbulent flows. However, the linear theory alone probably does not say much about the scale selection in the streamwise direction. In self-sustained processes, both the linear amplification and the nonlinear refuelling of the amplification process are necessary to select specific structures. The modelling of these additional nonlinear processes is completely absent from the present investigation. The exploration of these additional processes in fully turbulent flows is the object of current intensive investigation.

\subsection{Relevance of the harmonic forcing analysis}

Even if information on the spatio-temporal energy spectral densities for stochastic forcing can be extracted from the operator $\left[\boldsymbol{H}^{\dagger}\right](\alpha, \beta, \omega)$, the main use of the harmonic forcing analysis is in its prediction of the optimal response to deterministic forcing. The deterministic forcing analysis has, for instance, been recently used by McKeon (2008) to analyse the effect of 'dynamic roughness' at $R e_{\tau}=500$.

Our results indicate that the largest response to forcing is expected for the steady or very-low-frequency forcing of large-scale structures with $\lambda_{z} \approx 2-6 h$ and that the associated amplification can be very large, if compared to stochastic forcing, for example. A large sensitivity to steady forcing has indeed been observed in recent experiments on Couette and boundary layer flows (Kitoh et al. 2005; Kitoh \& Umeki 2008; Pujals, Cossu \& Depardon 2010a) where the large-scale streaky motions are artificially forced by passive devices.

The strong amplification of the harmonic forcing can be exploited to efficiently manipulate the turbulent mean flow. As the forced streaks must reach a finite amplitude in order to modify the mean flow at leading order, nonlinear effects must in principle be taken into account in the analysis. However, a series of previous investigations has shown that if the amplitude of the forced streaks is not too large $\left(\approx 10-15 \%\right.$ of $\left.U_{e}\right)$, the main nonlinear effects essentially reduce to a saturation of the streaks' amplitude, whose amplification, however, remains large. Indeed, Willis, Hwang \& Cossu $(2009,2010)$ have forced large-scale linearly optimal $\alpha=0$ vortices, computed with the same approach used in this study, in a DNS of the turbulent pipe flow. A large amplification of the forcing is observed and the induced large-scale finite-amplitude streaks modify the flow at leading order and induce a reduction of the turbulent drag consistently with the results of Schoppa \& Hussain (1998) (plane channel flow, DNS) and Iuso et al. (2002) (boundary layer, experimental results).

The above results more generally confirm the conclusions obtained in related studies, where the framework is spatial transient growth, that the large amplifications associated with the coherent lift-up effect can be successfully exploited for flow control applications. In the experiments of Fransson et al. (2004), finite-amplitude streamwise streaks with the optimal spanwise wavelength predicted by the linear optimal spatial transient growth analysis of Andersson, Berggren \& Henningson (1999) have been artificially forced in the laminar Blasius boundary layer. These streaks display large spatial amplifications, with amplitudes up to $\approx 13 \%$ of $U_{e}$, and the leading-order effect on the base flow is instrumental in improving its stability with respect to TollmienSchlichting waves (Cossu \& Brandt 2002, 2004; Fransson et al. 2005) and in delaying 
transition to turbulence (Fransson et al. 2006). In a similar way, Pujals et al. (2010a) have forced steady large-scale coherent streamwise streaks in the flat-plate turbulent boundary layer, also showing that in this case the large-scale coherent streaks display a non-normal amplification at finite amplitude. Furthermore, Pujals et al. (2010a) show that the most amplified spanwise wavelengths correspond well to the optimal values predicted in the optimal transient growth analysis of Cossu et al. (2009) despite the fact that this latter prediction is based on a temporal analysis (for $\alpha=0$ ) and not on a spatial analysis. Pujals, Depardon \& Cossu (2010b) have used the same type of forcing of Pujals et al. (2010a) to induce coherent streaks on the roof of an Ahmed body, which is relevant for industrial applications. In this latter case the forced streaks are no more optimal and the base flow is strongly non-parallel, but nevertheless the streaks are sufficiently amplified to modify the base flow at leading order. This mean flow modification has been shown to suppress the separation on the rear end of the Ahmed body.

\section{Summary and conclusions}

The main objective of the present investigation was to understand how small coherent perturbations to the turbulent channel flow respond to stochastic and deterministic forcing. Following Reynolds \& Hussain (1972), del Álamo \& Jiménez (2006), Pujals et al. (2009), Cossu et al. (2009) and Hwang \& Cossu (2010), we have used a linear model including eddy viscosity for the dynamics of coherent perturbations. The main results are as follows.

(a) Only structures elongated in the streamwise direction are significantly amplified, and the most amplified ones are streamwise uniform $\left(\alpha=0 ; \lambda_{x}=\infty\right)$. The optimal outputs are strongly anisotropic, with a dominant streamwise component (streaks), while the optimal inputs have dominant cross-stream components (streamwise vortices).

(b) The energy amplification by the optimal harmonic forcing is more than two orders of magnitude larger than the amplification of the variance induced by stochastic forcing.

(c) The amplification of the optimal harmonic and stochastic forcing of streamwise uniform structures are found to approximatively scale with the spanwise wavenumber as $\beta^{-2}$ and $\beta^{-1}$, respectively, in an intermediate $\beta$ range where the optimal structures are found to be, to a first approximation, geometrically similar.

(d) We show that the numerically observed $\beta^{-2}$ and $\beta^{-1}$ approximate scalings can be formally derived from the linearized system assuming geometric similarity for the coherent perturbations and a logarithmic base flow. Following this approach, it is also shown that, in the same regime, the optimal transient growth of initial perturbation does not depend on $\beta$ and that the time of maximum growth scales linearly with $\lambda_{z}$.

(e) To detect the deviations from the log-layer regime, the optimal harmonic $\left(R_{\max }\right)$ and stochastic $(V)$ amplifications obtained for $\alpha=0$ have been pre-multiplied by $\beta^{2}$ and $\beta$, respectively. The pre-multiplied amplification curves clearly show two peaks. For both the harmonic and the stochastic forcing cases, the dominant (outer) peak scales in outer units with a most-amplified wavelength of $\lambda_{z}=3.5 \mathrm{~h}$, while the secondary peak scales in inner units with an optimal wavelength of $\lambda^{+}=80$.

(f) The amplifications associated with the outer (dominant) peak increase with the Reynolds number, while those associated with the inner (secondary) peak and those in the log-layer scaling region are almost independent of the Reynolds number if scaled in inner units. 
( $g$ ) The shapes of the optimal input and outputs associated to the outer and inner peaks of the pre-multiplied amplifications are almost independent from the Reynolds number when plotted in outer and inner units, respectively. Furthermore, the optimal inputs and outputs of the three different optimal frameworks (harmonic forcing, stochastic forcing, initial-value problems) all have the same shapes. The profiles of the streak amplitude $\hat{u}(y)$ corresponding to the outer peak are proportional to the turbulent mean flow profile in the inner layer.

These results extend and complete the previous analyses of del Álamo \& Jiménez (2006) and Pujals et al. (2009), which considered the optimal response to initial conditions in the turbulent channel. In accordance with these studies we find that the optimal inputs and outputs are streamwise uniform and are quasi-geometrically similar in the intermediate $\beta$ range where they scale with their spanwise wavelength, which is also in accordance with the idea of geometrically similar attached eddies proposed by Townsend (1976). The two peaks in the 'pre-multiplied' amplification curves are also found at wavelengths $\lambda_{z}^{+} \approx 80-100$ and $\lambda_{z} / h \approx 3-4$ very similar to those found for the optimal initial-value problem. Optimal growths and perturbations with $\alpha \ll \beta$ are very similar to those found for $\alpha=0$. The optimal structures associated to the inner and outer peaks (possibly with $\alpha \ll \beta$ ) correspond qualitatively, in size and shape, to the most energetic streamwise streaks, respectively, observed in the buffer layer (Kline et al. 1967; Smith \& Metzler 1983; Robinson 1991) and at very large scales in the channel (del Álamo et al. 2004; Jiménez 2007), as already discussed by del Álamo \& Jiménez (2006) for example. This correspondence can only be qualitative because of the crude assumptions used in the present analysis, like the linear approximation, or the modelling of the Reynolds stress with an isotropic eddy viscosity.

The near-coincidence of the optimal perturbations pertaining to the three different analyses (initial-value problem, harmonic and stochastic forcing) is, however, unexpected and currently unexplained. This issue certainly deserves further investigation to understand whether this result is general and to provide a sound explanation for it.

The most important difference between the three different linear input-output analyses comes from the different scaling of the optimal amplifications in the intermediate $\beta$ range: $\beta^{-2}$ for the optimal harmonic forcing, $\beta^{-1}$ for the stochastic forcing and $\beta^{0}$ (no dependence) for the initial-value problem. We have shown how this different behaviour directly stems from the $\beta$ scaling of the whole linear generalized Orr-Sommerfeld-Squire operator obtained when perturbations are assumed to be geometrically similar and a base flow profile and eddy viscosity with log-layer properties are assumed. The same arguments explain the proportionality to $\lambda_{z}$ of the time of maximum temporal growth of log-layer structures observed by del Álamo \& Jiménez (2006) and Pujals et al. (2009).

Discussions with Dr A. P. Willis are gratefully acknowledged. Y.H. acknowledges funding from the French Ministry of Foreign Affairs through a Blaise Pascal Scholarship and from École Polytechnique through a Gaspard Monge Scholarship. Partial support by DGA is also kindly acknowledged.

\section{Appendix. Re-scaled operators in the log-layer approximation}

The Fourier amplitudes of spanwise periodic, geometrically similar streamwise uniform fields are given by the same function of the re-scaled wall-normal coordinate 
$\check{y}=\bar{\beta}(y+h)$, where $\check{\beta}$ is taken as the reference spanwise wavenumber, and the wavenumber re-scaling factor is defined by $\bar{\beta}=(\beta / \check{\beta})$. The chain rule immediately gives $\mathscr{D}=\bar{\beta} \check{\mathscr{D}}$ with $\check{\mathscr{D}}=\partial / \partial \check{y}$, and therefore $\Delta=\bar{\beta}^{2} \check{\Delta}$ and $\Delta^{-1}=\bar{\beta}^{-2} \check{\Delta}^{-1}$ with $\check{\Delta}=\check{\mathscr{D}}^{2}-\check{\beta}^{2}$.

As in the log layer the shear of the base flow is $U^{\prime}=u_{\tau} / \kappa(y+h)=\bar{\beta} u_{\tau} / \kappa \check{y}$, we assume that $U^{\prime}=\bar{\beta} \check{U}^{\prime}$, where $\check{U}^{\prime}=\check{\mathscr{D}} U(\check{y})$. Furthermore, in the log region the Reynolds stress is to a first approximation constant and equal to $u_{\tau}^{2}$, which gives for the eddy viscosity introduced in $\S 2 v_{T}(y)=u_{\tau} \kappa(y+h)=\bar{\beta}^{-1} u_{\tau} \kappa \check{y}$. We therefore also assume that $v_{T}=\bar{\beta}^{-1} \breve{v}_{T}$, where $\check{v}_{T}=v_{T} \check{y}$. From the above assumptions replaced in system (2.3) and further assuming $\alpha=0$, the following re-scaled system is found:

$$
\frac{\partial \check{\boldsymbol{q}}}{\partial t}=\bar{\beta} \check{\boldsymbol{A}} \check{\boldsymbol{q}}+\check{\boldsymbol{B}} \hat{\boldsymbol{f}}
$$

where $\check{\boldsymbol{q}}=\left[\check{v}, \check{\omega}_{y}\right]^{\mathrm{T}}$ with $\check{v}=\hat{v}, \check{\omega}_{y}=\hat{\omega}_{y} / \bar{\beta}$. As already mentioned in $\S 2.1$, the wallnormal vorticity had been re-scaled with $\bar{\beta}$ to preserve the self-similarity on the velocity field $\boldsymbol{u}$ when $\beta$ is changed. The operators $\check{\boldsymbol{A}}$ and $\breve{\boldsymbol{B}}$ are

$$
\check{\boldsymbol{A}}=\left[\begin{array}{cc}
\check{\Delta}^{-1} \check{\mathscr{L}}_{O \mathscr{S}} & 0 \\
-\mathrm{i} \check{\beta} \check{U}^{\prime} & \check{\mathscr{L}}_{\mathscr{S} \mathscr{Q}}
\end{array}\right], \quad \check{\boldsymbol{B}}=\left[\begin{array}{ccc}
0 & -\check{\beta}^{2} \check{\Delta}^{-1} & -\mathrm{i} \check{\beta} \check{\Delta}^{-1} \check{\mathscr{D}} \\
\mathrm{i} \check{\beta} & 0 & 0
\end{array}\right],
$$

with

$$
\begin{aligned}
& \check{\mathscr{L}}_{O \mathscr{S}}=\check{\mathrm{v}}_{T} \check{\Delta}^{2}+2 \check{\mathrm{v}}_{T}^{\prime} \check{\Delta} \check{\mathscr{D}}+\check{\mathrm{v}}_{T}^{\prime \prime}\left(\check{\mathscr{D}}^{2}+\check{\boldsymbol{\beta}}^{2}\right), \\
& \check{\mathscr{L}}_{\mathscr{S} \mathscr{Q}}=\check{v}_{T} \check{\Delta}+\check{v}_{T}^{\prime} \check{\mathscr{D}} \text {. }
\end{aligned}
$$

The velocity components are obtained from the wall-normal variables with

$$
\hat{\boldsymbol{u}}=\check{\mathbf{C}} \check{\boldsymbol{q}}, \quad \check{\boldsymbol{C}}=\frac{1}{\check{\beta}^{2}}\left[\begin{array}{cc}
0 & -\mathrm{i} \check{\beta} \\
\check{\beta}^{2} & 0 \\
\mathrm{i} \check{\beta} \mathscr{D} & 0
\end{array}\right] .
$$

The re-scaled state vector $\check{\boldsymbol{q}}$ can also be obtained from the velocity with

$$
\check{\boldsymbol{q}}=\check{\boldsymbol{D}} \hat{\boldsymbol{u}}, \quad \check{\boldsymbol{D}}=\left[\begin{array}{ccc}
0 & 1 & 0 \\
\mathrm{i} \check{\beta} & 0 & 0
\end{array}\right] .
$$

The operators $\check{\boldsymbol{A}}, \check{\boldsymbol{B}}, \check{\boldsymbol{C}}, \check{\boldsymbol{D}}$ and $\check{\mathscr{L}}_{\mathcal{O} \mathscr{S}}, \check{\mathscr{L}}_{\mathscr{S} \mathscr{Q}}$ do not depend on $\bar{\beta}$ (and $\beta$ ), but only on the reference value $\breve{\beta}$.

\section{REFERENCES}

DEL Álamo, J. \& JimÉnez, J. 2003 Spectra of the very large anisotropic scales in turbulent channels. Phys. Fluids 15, L41.

Del Álamo, J. C. \& JimÉnez, J. 2006 Linear energy amplification in turbulent channels. J. Fluid Mech. 559, 205-213.

del Álamo, J. C., Jiménez, J., Zandonade, P. \& Moser, R. D. 2004 Scaling of the energy spectra of turbulent channels. J. Fluid Mech. 500, 135-144.

Andersson, P., Berggren, M. \& Henningson, D. 1999 Optimal disturbances and bypass transition in boundary layers. Phys. Fluids 11 (1), 134-150.

BAmien, B. \& DAhleh, M. 2001 Energy amplification in channel flows with stochastic excitation. Phys. Fluids 13, 3258-3269.

Butler, K. M. \& Farrell, B. F. 1992 Three-dimensional optimal perturbations in viscous shear flow. Phys. Fluids A 4, 1637-1650. 
CEss, R. D. 1958 A survey of the literature on heat transfer in turbulent tube flow. Res. Rep. 8-0529-R24. Westinghouse.

Cossu, C. \& BrandT, L. 2002 Stabilization of Tollmien-Schlichting waves by finite amplitude optimal streaks in the Blasius boundary layer. Phys. Fluids 14, L57-L60.

Cossu, C. \& BrandT, L. 2004 On Tollmien-Schlichting waves in streaky boundary layers. Eur. J. Mech. B/ Fluids 23, 815-833.

Cossu, C., Pujals, G. \& Depardon, S. 2009 Optimal transient growth and very large scale structures in turbulent boundary layers. J. Fluid Mech. 619, 79-94.

Ellingsen, T. \& Palm, E. 1975 Stability of linear flow. Phys. Fluids 18, 487.

Farrell, B. F. \& IOANnOU, P. J. $1993 a$ Optimal excitation of three-dimensional perturbations in viscous constant shear flow. Phys. Fluids 5, 1390-1400.

Farrell, B. F. \& IoANnou, P. J. $1993 b$ Stochastic forcing of the linearized Navier-Stokes equation. Phys. Fluids A 5, 2600-2609.

FArRell, B. F. \& IOANnoU, P. J. 1994 Variance maintained by the stochastic forcing of non-normal dynamical systems associated with linearly stable shear flows. Phys. Rev. Lett. 72, 11881191.

Farrell, B. F. \& Ionnnou, P. J. 1996 Generalized stability theory. J. Atmos. Sci. 53, 20252053.

Farrell, B. F. \& IoAnnou, P. J. 1998 Perturbation structure and spectra in turbulent channel flow. Theor. Comput. Fluid Dyn. 11, 237-250.

Fransson, J., Brandt, L., Talamelli, A. \& Cossu, C. 2004 Experimental and theoretical investigation of the non-modal growth of steady streaks in a flat plate boundary layer. Phys. Fluids 16, 3627-3638.

Fransson, J., Brandt, L., Talamelli, A. \& Cossu, C. 2005 Experimental study of the stabilisation of Tollmien-Schlichting waves by finite amplitude streaks. Phys. Fluids 17, 054110.

Fransson, J., Talamelli, A., Brandt, L. \& Cossu, C. 2006 Delaying transition to turbulence by a passive mechanism. Phys. Rev. Lett. 96, 064501.

De GraffF, D. \& Eaton, J. 2000 Reynolds-number scaling of the flat-plate turbulent boundary layer. J. Fluid Mech. 422, 319-346.

Guala, M., Hommema, S. E. \& Adrian, R. J. 2006 Large-scale and very large-scale motions in turbulent pipe flow. J. Fluid Mech. 554, 521-541.

Gustavsson, L. H. 1991 Energy growth of three-dimensional disturbances in plane Poiseuille flow. J. Fluid Mech. 224, 241-260.

Hamilton, J., Kim, J. \& Waleffe, F. 1995 Regeneration mechanisms of near-wall turbulence structures. J. Fluid Mech. 287, 317-348.

Hoyas, S. \& JimÉnez, J. 2006 Scaling of the velocity fluctuations in turbulent channels up to $R e_{\tau}=2003$. Phys. Fluids 18, 011702.

Hunt, J. C. R. \& Morrison, J. F. 2000 Eddy structure in turbulent boundary layer. Eur. J. Mech. B 19, 673-694.

Hutchins, N. \& Marusic, I. 2007a Evidence of very long meandering features in the logarithmic region of turbulent boundary layers. J. Fluid Mech. 579, 1-28.

Hutchins, N. \& Marusic, I. $2007 b$ Large-scale influences in near-wall turbulence. Phil. Trans. R. Soc. A 365, 647-664.

Hwang, Y. \& Cossu, C. 2010 Amplification of coherent streaks in the turbulent Couette flow: an input-output analysis at low Reynolds number. J. Fluid Mech. 643, 333-348.

Iuso, G., Onorato, M., Spazzini, P. G. \& Di Cicca, G. M. 2002 Wall turbulence manipulation by large-scale streamwise vortices. J. Fluid Mech. 473, 23-58.

JimÉNEZ, J. 1998 The largest scales of turbulent wall flows. In CTR Annual Research Briefs, pp. 943-945. Center for Turbulence Research, Stanford University.

JimÉNEZ, J. 2007 Recent developments on wall-bounded turbulence. Rev. R. Acad. Cien. Ser. A Mat. 101, 187-203.

JiméNEZ, J. \& Morn, P. 1991 The minimal flow unit in near-wall turbulence. J. Fluid Mech. 225, 213-240.

JimÉnez, J. \& Pinelli, A. 1999 The autonomous cycle of near-wall turbulence. J. Fluid Mech. 389, 335-359.

Jovanović, M. R. \& Bamien, B. 2005 Componentwise energy amplification in channel flow. J. Fluid Mech. 543, 145-83. 
Kim, K. C. \& Adrian, R. 1999 Very large-scale motion in the outer layer. Phys. Fluids 11 (2), $417-422$.

Kitoh, O., Nakabayashi, K. \& Nishimura, F. 2005 Experimental study on mean velocity and turbulence characteristics of plane Couette flow: low-Reynolds-number effects and large longitudinal vortical structures. J. Fluid Mech. 539, 199.

Кітон, O. \& Uмекi, M. 2008 Experimental study on large-scale streak structure in the core region of turbulent plane Couette flow. Phys. Fluids 20, 025107.

Kline, S. J., Reynolds, W. C., Schraub, F. A. \& Runstadler, P. W. 1967 The structure of turbulent boundary layers. J. Fluid Mech. 30, 741-773.

Komminaho, J., Lundbladh, A. \& Johansson, A. V. 1996 Very large structures in plane turbulent Couette flow. J. Fluid Mech. 320, 259-285.

LANDAHL, M. T. 1980 A note on an algebraic instability of inviscid parallel shear flows. J. Fluid Mech. 98, 243-251.

LANDAHL, M. T. 1990 On sublayer streaks. J. Fluid Mech. 212, 593-614.

Malkus, W. V. R. 1956 Outline of a theory of turbulent shear flow. J. Fluid Mech. 1, 521-539.

Mathis, R., Hutchins, N. \& Marusic, I. 2009 Large-scale amplitude modulation of the small-scale structures in turbulent boundary layers. J. Fluid. Mech. 628, 311-337.

McKeon, B. J. 2008 A model for 'dynamic' roughness in turbulent channel flow. In Proceedings of the Summer Program, pp. 399-410. Center for Turbulence Research, Stanford University.

Metzger, M. M. \& KLewicki, J. C. 2001 A comparative study of near-wall turbulence in high and low Reynolds number boundary layers. Phys. Fluids 13, 692-701.

MoffatT, H. K. 1967 The interaction of turbulence with a strong wind shear. In Proceedings URSIIUGG International Colloquium on Atmospheric Turbulence and Radio Wave Propagation, pp. 139-154. Nauka.

Morrison, W. R. B. \& Kronauer, R. E. 1969 Structural similarity for fully developed turbulence in smooth tubes. J. Fluid Mech. 39, 117-141.

Perry, A. E. \& Chong, M. S. 1982 On the mechanism of turbulence. J. Fluid Mech. 119, 173-217.

Pujals, G., Cossu, C. \& Depardon, S. 2010a Forcing large-scale coherent streaks in a zero pressure gradient turbulent boundary layer. J. Turbulence 11 (25), 1-13.

Pujals, G., Depardon, S. \& Cossu, C. $2010 b$ Drag reduction of a 3D bluff body using coherent streamwise streaks. Exp. Fluids (in press).

Pujals, G., García-Villalba, M., Cossu, C. \& Depardon, S. 2009 A note on optimal transient growth in turbulent channel flows. Phys. Fluids 21, 015109.

Reddy, S. C. \& Henningson, D. S. 1993 Energy growth in viscous channel flows. J. Fluid Mech. 252, 209-238.

Reddy, S. C., Schmid, P. J. \& Henningson, D. S. 1993 Pseudospectra of the Orr-Sommerfeld operator. SIAM J. Appl. Math. 53, 1547.

Reynolds, W. C. \& Hussain, A. K. M. F. 1972 The mechanics of an organized wave in turbulent shear flow. Part 3. Theoretical models and comparisons with experiments. J. Fluid Mech. 54 (2), 263-288.

Reynolds, W. C. \& Tiederman, W. G. 1967 Stability of turbulent channel flow, with application to Malkus's theory. J. Fluid Mech. 27 (2), 253-272.

Robinson, S. K. 1991 Coherent motions in the turbulent boundary layer. Annu. Rev. Fluid Mech. 23, 601-639.

SCHmid, P. 2007 Nonmodal stability theory. Annu. Rev. Fluid Mech. 39, 129-162.

Schmid, P. \& Henningson, D. S. 1994 Optimal energy density growth in Hagen-Poiseuille flow. J. Fluid Mech. 277, 197-225.

Schmid, P. J. \& Henningson, D. S. 2001 Stability and Transition in Shear Flows. Springer.

Schoppa, S. \& Hussain, F. 1998 A large-scale control strategy for drag reduction in turbulent boundary layers. Phys. Fluids 10, 1049.

Schoppa, W. \& Hussain, F. 2002 Coherent structure generation in near-wall turbulence. J. Fluid Mech. 453, 57-108.

Smith, C. R. \& Metzler, S. P. 1983 The characteristics of low-speed streaks in the near-wall region of a turbulent boundary layer. J. Fluid Mech. 129, 27-54.

Tomkins, C. D. \& Adrian, R. J. 2003 Spanwise structure and scale growth in turbulent boundary layers. J. Fluid Mech. 490, 37-74. 
Tomkins, C. D. \& Adrian, R. J. 2005 Energetic spanwise modes in the logarithmic layer of a turbulent boundary layer. J. Fluid Mech. 545, 141-162.

Townsend, A. 1976 The Structure of Turbulent Shear Flow, 2nd edn. Cambridge University Press.

Trefethen, L. N., Trefethen, A. E., Reddy, S. C. \& Driscoll, T. A. 1993 A new direction in hydrodynamic stability: beyond eigenvalues. Science 261, 578-584.

WALEFFE, F. 1995 Hydrodynamic stability and turbulence: beyond transients to a self-sustaining process. Stud. Appl. Math. 95, 319-343.

Weideman, J. A. C. \& Reddy, S. C. 2000 A MATlab Differentiation Matrix Suite. ACM Trans. Math. Softw. 26, 465-519.

Willis, A. P., Hwang, Y. \& Cossu, C. 2009 Optimal harmonic forcing and drag reduction in pipe flow. Bull. Am. Phys. Soc. 54 (19), 227.

Willis, A. P., Hwang, Y. \& Cossu, C. 2010 Optimally amplified large-scale streaks and drag reduction in the turbulent pipe flow. Phys. Rev. E (submitted).

Zhou, K., Doyle, J. \& Glover, K. 1996 Robust and Optimal Control. Prentice-Hall. 